\title{
Activation of the Integrated Stress Response overcomes multidrug resistance in FBXW7-deficient cells
}

Laura Sanchez-Burgos ${ }^{1 *}$, Belén Navarro-González¹, Santiago García-Martín², Héctor Tejero², Marta Elena Antón ${ }^{1}$, Fátima Al-Shahrour² \& Oscar Fernandez-Capetillo ${ }^{1,3}$

1 Genomic Instability Group, Spanish National Cancer Research Centre (CNIO), 28029 Madrid, Spain.

2 Bioinformatics Unit, Spanish National Cancer Research Centre (CNIO), 28029 Madrid, Spain.

3 Science for Life Laboratory, Division of Genome Biology, Department of Medical Biochemistry and Biophysics, Karolinska Institute, S-171 21 Stockholm, Sweden.

Lead Contact: $\quad$ Oscar Fernandez-Capetillo

Spanish National Cancer Research Centre (CNIO)

Melchor Fernandez Almagro, 3

Madrid 28029, Spain

Tel.: $\quad+34.91 .732 .8000$ Ext: 3480

Fax: $\quad+34.91 .732 .8028$

Email: ofernandez@cnio.es

Twitter: @KP Twitt Ilo 


\section{SUMMARY}

$F B X W 7$ is one of the most frequently mutated tumor suppressors, the deficiency of which has been associated with resistance to some anticancer therapies. Through bioinformatic analyses and genome-wide CRISPR screens, we here reveal that FBXW7 deficiency leads to multidrug resistance (MDR), to a bigger extent than well-established MDR-drivers such as overexpression of the drug-efflux pump ABCB1. Proteomic data from FBXW7-deficient cancer cells identify the upregulation of mitochondrial function as a hallmark of FBXW7 deficiency, which has been previously linked to an increased resistance to chemotherapy. Accordingly, genetic or chemical targeting of mitochondria is preferentially toxic for FBXW7-deficient cells in vitro and in vivo. Mechanistically, we show that the toxicity associated with therapies that target mitochondrial translation such as the antibiotic tigecycline relates to the activation of the Integrated Stress Response (ISR). Furthermore, while searching for additional drugs that could overcome the MDR of FBXW7-deficient cells, we found that all of them unexpectedly also activated the ISR regardless of their currently accepted mechanism of action. Together, our study reveals that one of the most frequent mutations in cancer reduces the sensitivity to the vast majority of available therapies, and identifies a general principle to overcome such resistance. 


\section{INTRODUCTION}

Resistance to therapy has been estimated to contribute to treatment failure in up to $90 \%$ of cancer patients and remains one of the fundamental challenges in cancer ${ }^{1,2}$. This is also true in the context of immune therapies, the efficiency of which is limited by mutations that reduce antigen presentation or inflammatory signaling ${ }^{3}$. Accordingly, "to develop ways to overcome cancer's resistance to therapy" was one of the 10 recommendations made from the Blue Ribbon Pannel associated with the Cancer Moonshot initiative of the National Cancer Institute ${ }^{4}$. Starting in the $1960 \mathrm{~s}^{5}$, intensive research has led to the identification of multiple mutations involved in the resistance to single-agents, leading to the development of targeted therapies that can overcome the resistance. Examples of this are the discovery of MEK reactivation in RAF inhibitortreated BRAF-mutant melanoma cells, or the emergence of drug-resistant mutations in EGFR in lung cancer, both of which have led to new treatment strategies with improved success $^{6,7}$. Unfortunately, cancer patients under treatment often acquire multidrug resistance $(M D R)^{8}$, which greatly limits their subsequent therapeutic opportunities.

Two of the best known mediators of MDR are the activation of efflux pumps such as $A B C B 1$ that limit intracellular drug concentrations ${ }^{9}$ and the dysregulation of the intrinsic apoptotic pathway through the upregulation of anti-apoptotic proteins like MCL1 ${ }^{10}$. Besides the contribution of specific genetic determinants, several evidences indicate that phenotypic changes can also modify the response to therapy in cancer cells. Examples of this include the epithelial-to-mesenchymal transition (EMT) ${ }^{11}$, transdifferentiation into another cell type ${ }^{12}$ or entering into a diapause-like state ${ }^{13}$, all of which have been shown to lead to an increased resistance to chemotherapy. In this regard, an enhanced mitochondrial activity has recently been associated with the resistance to several individual agents ${ }^{14-23}$ as well as to $\mathrm{MDR}^{24-26}$. Accordingly, targeting mitochondrial function has emerged as an interesting therapeutic opportunity to overcome drug resistance ${ }^{27,28}$. Furthermore, some tumors such as acute myeloid leukemia $(A M L)^{29}$, B-RAF driven melanomas ${ }^{26}$ or C-MYC driven lymphomas ${ }^{30}$ are specifically dependent on mitochondrial translation, which renders them sensitive to certain antibiotics such as tigecycline that 
affect the function of eukaryotic ribosomes due to their structural resemblance to those from bacteria 31,32 .

FBXW7 is the substrate receptor component of the Skp1-Cdc53/Cullin-F-box-protein (SCF) ubiquitin ligase complex, which mediates the degradation of important oncoproteins such as Cyclin E1 (CCNE1) ${ }^{33}, \mathrm{MYC}^{34}$, JUN ${ }^{35}$ and $\mathrm{NOTCH}^{36}$ upon their phosphorylation on CDC4 phosphodegron (CPD) domains. In fact, FBXW7 is one of the 10 most frequently mutated genes in human cancers ${ }^{37}$, due to either inactivating mutations and/or allelic loss 38,39 . Moreover, mutations in FBXW7 are amongst the most significantly associated with poor survival across all human cancers ${ }^{40}$. Besides its oncogenic potential, loss of FBXW7 has also been linked to an increased resistance to various chemotherapies ${ }^{41}$ and immunotherapy ${ }^{42}$. Furthermore, forward genetic screens have found an enrichment of FBXW7 mutations among those that drive resistance to various anticancer agents ${ }^{43-46}$. While early works indicated that the increased resistance of FBXW7-deficient cells to agents such as Taxol was due to the stabilization of the antiapoptotic factor $\mathrm{MCL}^{47}$, other mechanisms such as the induction of an EMT have been also proposed to modulate drug sensitivities in FBXW7-mutant tumors ${ }^{48}$. We here systematically addressed the impact of FBXW7 deficiency in the response to anticancer therapies, and identify a general strategy to overcome therapy resistance in FBXW7deficient cancer cells. 


\section{RESULTS}

\section{FBXW7 deficiency leads to multidrug resistance}

We previously generated mouse embryonic stem cells (mES) harboring a doxycycline-inducible Cas9 and used them to conduct forward genetic screens in order to identify the mechanisms of resistance to inhibitors of the ATR kinase ${ }^{49}$. Following the same pipeline (described in Fig. S1A), we performed genetic screens to identify mutations that confer resistance to various cytotoxic agents such as cisplatin, rigosertib or ultraviolet light (UV). When analyzing the sgRNAs present in clones of mutant ES that had become resistant to these treatments, we noted a high frequency of sgRNAs targeting Fbxw7 (Fig. S1B). A similar enrichment of Fbxw7-targeting sgRNAs was observed in pools of treatment-resistant mutagenized ES populations that were analyzed by sequencing (Fig. S1C). Given the previous literature linking FBXW7 mutations to resistance to various cancer therapies, we wondered to what extent these observations were reflecting a more general phenomenon and whether FBXW7 deficiency could lead to MDR.

To test the impact of FBXW7 deficiency in the response to cancer therapies, we generated Fbxw7 wild type (WT) and knockout mES cell lines by CRISPR editing (Fig. S2A) which constitutively expressed the fluorescent proteins EGFP or RUBY3, respectively. We then evaluated the effect of different drugs in co-cultures of $\mathrm{FbxW7}^{+/+}$ and $F b x w 7^{-1}$ mES by monitoring the evolution in the percentages of EGFP- and RUBY3positive cells by FACS. Consistent with the reported resistance of FBXW7-mutant human cancer cells to paclitaxel ${ }^{47,50}$, oxaliplatin ${ }^{51,52}, 5$-fluorouracil $(5-\mathrm{FU})^{53,54}$ and doxorubicin ${ }^{55}$, Fbxw $7^{-1-} \mathrm{mES}$ cells became significantly enriched after $48 \mathrm{hrs}$ of culture in the presence of these drugs (Fig. S2B). Next, and to systematically address the response of Fbxw7deficient cells to chemotherapy, we used the same $\mathrm{Fbxw} 7^{+/+} / \mathrm{Fb} \mathrm{F} w 7^{-I_{-}}$competition assay to evaluate a chemical library of 114 FDA-approved antitumoral compounds. Strikingly, this analysis revealed a strong selection for Fbxw7-deficient mES cells upon treatment with many different drugs, which was more pronounced with the compounds that had the highest toxicity (Fig. 1A,B). 
To confirm whether the widespread resistance to chemotherapy was also seen in human cancer cells, we generated $F B X W 7^{+/+}$and $F B X W 7^{-/-}$clones in the colorectal adenocarcinoma cell line DLD-1 (Fig. S2C). We chose colorectal carcinoma as this is the cancer type with the highest frequency of $F B X W 7$ mutations according to data available at The Cancer Genome Atlas (TCGA). Similar to our observations in mES, FBXW7-/-DLD1 cells were significantly resistant to 10 anticancer drugs with different mechanisms of action (Fig. 1C). To obtain a more general view of how FBXW7 deficiency impacts on the response to anticancer drugs, we interrogated data from the $\mathrm{NCl}-60$ repository, where the response of 60 different cancer cell lines to thousands of compounds is available together with genomic and transcriptomic data for each cell line ${ }^{56}$. Consistent with the known role of $\mathrm{ABCB} 1$ as a mediator of MDR, $A B C B 1$ mutant cells were resistant to the majority of the drugs tested in this dataset (Fig. 1D). Strikingly, this trend was even more accentuated in FBXW7-mutant cell lines. The MDR phenotype associated to FBXW7 mutations was also observed by interrogating the Cancer Cell Line Encyclopedia (CCLE), which contains data from 1,072 cell lines ${ }^{57}$ (Fig. 1E). Similar analyses performed on the Cancer Therapeutics Response Portal $(\mathrm{CTRP})^{58}$ revealed that the increased resistance to chemotherapy not only correlated with FBXW7 mutations but also with low mRNA expression (Fig. S2D), highlighting the potential of using FBXW7 levels as a general biomarker for drug responses. In support of this view, analysis of survival data from the Genomics Data Commons (GDC) portal ${ }^{59}$ revealed that low levels of FBXW7 expression significantly correlated with poor survival in cancer patients undergoing any type of therapy (Fig. S2E,F). Together, these experiments provide compelling evidence indicating that FBXW7 deficiency leads to a profound MDR phenotype in human cancer cells.

\section{MCL1 and ABCB1 independently contribute to drug resistance in FBXW7-deficient cells}

Given that MCL1 is a FBXW7 target that has been previously shown to contribute to the resistance to certain agents such as paclitaxel ${ }^{47}$, we evaluated if this could explain 
the MDR phenotype of FBXW7-mutant cells. To do so, we generated MCL1 knockouts in $F B X W 7^{+/+}$and $F B X W 7^{-/-}$DLD-1 cells by CRISPR editing (Fig. S3A) and tested their response to the same 10 drugs that we previously observed were less toxic for FBXW7deficient cells (Fig. 1C). While MCL1 deficiency was able to partly overcome the resistance of FBXW7-/- DLD-1 cells to 4 of these drugs, including paclitaxel, it had no significant effect in the other six compounds (Fig. S3B). Following a similar strategy, we tested whether deletion of the drug-efflux pump ABCB1 could revert the MDR of FBXW7deficient cells. Once again, while ABCB1 deficiency was able to significantly sensitize FBXW7-/- DLD-1 cells to 6 of the drugs, it had no impact on the others (Fig. S3C,D). Importantly, neither MCL1 nor ABCB1 loss were able to overcome the resistance of FBXW $7^{-/-}$cells to some of the compounds such as Trametinib, 5-FU or Gemcitabine. Together, these data imply that while specific factors such as ABCB1 or MCL1 might contribute to the resistance of FBXW7-deficient cells to some chemotherapies, another mechanism must account for the more general MDR phenotype found in these cells.

\section{FBXW7-deficient cells and tumors present an increase in mitochondrial pathways}

To investigate whether phenotypic changes could contribute to the MDR of FBXW7deficient cells, we compared the proteomes of FBXW7 WT and knockout DLD-1 and mES cells. Known targets of FBXW7 such as MYC, DAB2IP or MED13 were upregulated in FBXW7-deficient cells in both cell types (Fig. 2A). Besides individual factors, gene set enrichment analyses (GSEA) from proteins that showed increased levels in FBXW7-- cells revealed a significant enrichment in pathways related to mitochondria (Fig. 2B), "mitochondrial translation" showing the highest enrichment (Fig. 2C). Western blot and immunofluorescence experiments revealed an increase in mitochondrial volume and in levels of complexes from the oxidative phosphorylation (OXPHOS) pathway in $F B X W 7^{-/-}$ cells (Fig. S4). A similar enrichment of mitochondrial pathways was seen in Fbxw $7^{-1-}$ mES cells (Fig. 2D,E), and comparative analyses confirmed a generalized increase in the levels of mitochondrial proteins in both cell types (Fig. 2F). Finally, we used proteomic data available at the CCLE to investigate if equivalent observations were also seen in 388 cancer cell lines. In fact, these analyses confirmed a significant enrichment of 
mitochondrial proteins in FBXW7-mutant cell lines (Fig. 2G). In addition, GSEA analyses identified an enrichment of pathways related to mitochondria and oxidative phosphorylation in FBXW7-mutant cell lines from the CCLE panel (Fig. $\mathbf{2 H , I ) . ~ T o g e t h e r , ~}$ these data identify that increased mitochondrial activity is a hallmark of FBXW7-deficient cells.

\section{Targeting mitochondrial function is preferentially toxic for FBXW7-deficient cells}

As mentioned above, mitochondrial translation can be targeted by antibiotics that also inhibit the eukaryotic mitochondrial ribosome ${ }^{31,32}$. Competition experiments using $F B X W 7^{+/+}$and $F B X W 7^{-/-}$DLD-1 cells expressing EGFP and RUBY3, respectively, revealed that several antibiotics led to a depletion of $F B X W 7^{-/-}$cells, with tigecycline having the biggest effect (Fig. 3A,B). Counting nuclei by High-Throughput Microscopy (HTM) revealed that this effect was due to a preferential toxicity of tigecycline for FBXW7/- DLD-1 cells (Fig. 3C). A similar sensitivity to tigecycline was also seen in FBXW7deficient HeLa and A2780 cells generated by CRISPR, ruling out cell line-specific effects (Fig. S5). The preferential toxicity of tigecycline was also confirmed in tumor xenografts of FBXW7-1-DLD-1 cells, the growth of which was in contrast unaffected by paclitaxel (Fig. 3D,E). Of note, the sensitivity of FBXW7-deficient cells to tigecycline was partly rescued by siRNA-mediated depletion of the oncogene MYC, which is a FBXW7 target previously shown to confer sensitivity to the inhibition of mitochondrial translation ${ }^{30}$ (Fig. S6A,B). MYC depletion also reduced the levels of several mitochondrial factors in FBXW7-/- DLD1 cells (Fig. S6A). Besides antibiotics, drugs targeting OXPHOS such as IACS-1075960 or the mitochondrial F1F0 ATPase inhibitor oligomycin were also preferentially toxic for FBXW7-/- DLD-1 cells (Fig. 3F,G), yet not as much as tigecycline. Finally, and in agreement with the effects observed with chemicals, depletion of the mitochondrial factors TUFM, POLRMT, PTCD3, MRPS27 and UQCRC1 by enzymatically generated short interfering RNAs (esiRNA) was also preferentially toxic for FBXW7-/- DLD-1 cells as shown in competition experiments (Fig. $\mathbf{3 H}$ ). Together, these experiments demonstrate that targeting mitochondrial function is able to overcome the widespread resistance to chemotherapy associated to FBXW7 deficiency in cancer cells. 


\section{The toxicity of tigecycline is mediated by the integrated stress response}

Next, we aimed to understand the mechanism by which tigecycline promotes the preferential killing of FBXW7-deficient cells. To this end, we evaluated the transcriptional changes induced by tigecycline in $F B X W 7^{+++}$and FBXW7--- DLD-1 cells. Consistent with toxicity experiments, tigecycline had a significantly bigger impact on the transcriptome of FBXW7-1- cells (Fig. 4A). Gene Ontology (GO) analyses revealed that the antibiotic triggered various stress responses, including the endoplasmic reticulum (ER) stress response or the cellular response to arsenate, which were particularly acute in mutant cells (Fig. S7). These hallmarks suggested that tigecycline was activating the Integrated Stress Response (ISR), a signaling network that reprograms gene expression to respond to a wide range of insults but which can also promote apoptosis to eliminate the damaged cell ${ }^{61}$. In support of this, tigecycline promoted the nuclear accumulation of the transcription factor ATF4, a hallmark of the ISR (Fig. 4B) ${ }^{62}$. Furthermore, tigecycline-induced nuclear translocation of ATF4 was accentuated in FBXW7-1- cells, and was reverted by the ISR inhibitor ISRIB ${ }^{63,64}$ (Fig. 4B). More importantly, clonogenic survival assays revealed that ISRIB fully rescues the toxicity of tigecycline in both WT and FBXW7-deficient DLD-1 cells, confirming that the cytotoxic effects of the antibiotic are mediated by the ISR (Fig. $4 C)$.

\section{Drugs activating the ISR overcome the MDR of FBXW7-deficient cancer cells}

Finally, we sought to identify additional drugs that could overcome the widespread resistance to chemotherapy of FBXW7-deficient cells. To do so, we interrogated the Connectivity Map (CMap) dataset, which contains the transcriptional signatures triggered by thousands of drugs in cancer cells ${ }^{65}$, in order to identify drugs that elicit a transcriptional signature similar to that of tigecycline. Consistent with our RNAseq results, the signatures induced by the mitochondrial poison oligomycin and the ISR activators tunicamycin and salubrinal showed the highest similarity to that from tigecycline (Fig. 5A). In addition, and like tigecycline or oligomycin, $F B X W 7^{-1}$ cells were also sensitive to tunicamycin, confirming that activating the ISR is preferentially toxic for FBXW7-deficient cells (Fig. 5B). Interestingly, the list of drugs with signatures most similar to tigecycline included 
other drugs with seemingly distinct mechanisms of action such as B-RAF inhibitors (PLX4720 and vemurafenib), broad spectrum tyrosine kinase inhibitors (sorafenib and dasatinib) and EGFR inhibitors (erlotinib and gefitinib). Given the similarity of the transcriptional signatures triggered by these drugs to those of tigecycline and tunicamycin, we wondered if they also activated the ISR. In fact, all of these drugs promoted the nuclear accumulation of ATF4, which was reverted by ISRIB (Fig. 5C and Fig. S8A). In addition, these drugs promoted the accumulation of $\mathrm{CHOP}$, a transcription factor that mediates the apoptosis triggered by the ISR (Fig. S8B). As to whether these drugs were able to overcome the MDR of FBXW7-deficient cells, competition experiments

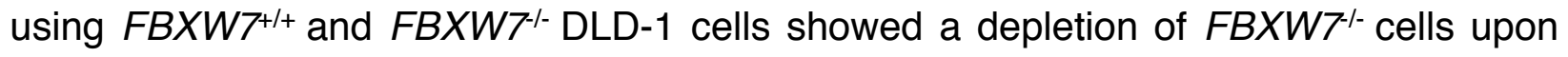
treatment with all of the drugs (Fig. 5D). Similarly, HTM-mediated quantification of nuclei confirmed that these compounds were preferentially toxic for FBXW7-- cells, in a manner that could be rescued by ISRIB (Fig. 5E). Noteworthy, drugs to which FBXW7-deficient cells are resistant such as paclitaxel or trametinib failed to activate the ISR as measured by the accumulation of ATF4 or CHOP (Fig. 5C and S8B).

Finally, we investigated which one of the 4 kinases that activate the ISR (HRI, GCN2, PERK or PKR) mediated the effects of these drugs on the ISR. To do so, we generated DLD-1 cells carrying a transcriptional reporter where a destabilized EGFP is placed under the control of the human CHOP promoter. These cells were subsequently infected with lentiviral viruses expressing mCherry and sgRNAs targeting ISR kinases or an empty vector. Flow cytometry analyses revealed that GCN2 deletion prevented the expression of CHOP triggered by tigecycline and by the drugs that overcome the MDR of FBXW7deficient cells (Fig. 5F). Furthermore, infection with lentiviruses expressing sgRNAs against GCN2, but not against HRI, PERK or PKR, reduced the toxic effect of all of these drugs (Fig. 5G). Collectively, these results identify the activation of the ISR as a general strategy to overcome drug resistance in FBXW7-deficient cancer cells, and suggest that the toxic effects of several anticancer drugs might be partly contributed by a previously unknown effect of the compounds in activating the GCN2-dependent branch of the ISR. 


\section{DISCUSSION}

We here show that FBXW7 deficiency, one of the most frequent events in human cancer ${ }^{37}$, increases the resistance to the vast majority of available anticancer therapies, likely contributing to the bad prognosis that is associated to FBXW7 mutations ${ }^{40}$. Since the increased resistance to chemotherapies is also associated to reduced $F B X W 7$ levels, this opens the possibility of using FBXW7 expression as a biomarker to predict an unfavorable response to treatment. We further reveal that the MDR is associated to an increased mitochondrial activity, rendering FBXW7-deficient tumors to reagents that target mitochondrial function. Noteworthy, our proteomic analysis is consistent with the analysis of transcriptional signatures from The Cancer Genome Atlas that indicate an increase in mitochondrial gene expression in FBXW7-deficient tumors ${ }^{66}$. As for the mechanism behind this phenomenon, several evidences indicate an important role for MYC. First, MYC is an FBXW7 target $^{34}$ that stimulates mitochondrial biogenesis and activity ${ }^{24,67}$. Second, increased levels of MYC have been shown to increase resistance to several therapies ${ }^{24,68}$. Finally, MYC overexpression renders cancer cells sensitive to targeting mitochondrial activity with antibiotics ${ }^{69}$ or drugs targeting $\mathrm{OXPHOS}^{70}$. Given the increasing availability of chemical- or peptide-based therapies for targeting $\mathrm{MYC}^{71}$, whether these agents might be useful as a general strategy to overcome the MDR in cancer emerges as an interesting possibility.

Targeting mitochondrial activity has been a long-debated approach in cancer therapy. Early clinical efforts faced either toxicities of drugs targeting OXPHOS or limited efficacy from tetracycline antibiotics ${ }^{27,28}$. Nevertheless, there is an intense preclinical development of additional chemotherapies that affect mitochondrial function which include inhibitors of mitochondrial transcription ${ }^{72}$, inhibitors of the METTL8 RNA methyltransferase ${ }^{73}$, and even the use of a diet low in valine, which has been recently shown to preferentially impair mitochondrial function and exert antitumoral properties ${ }^{74}$. Interestingly, a recent study also revealed that mitochondrial activity can be used to identify a glioblastoma subtype that is vulnerable to OXPHOS inhibitors ${ }^{75}$. Despite this renewed interest in targeting mitochondria in cancer, important questions still remain to 
be addressed. In our opinion, and before these strategies are brought to clinical trials, it would be important to identify the genetic determinants that modulate the response to these treatments, both to select patients that are most likely to respond but also to avoid their use in patients carrying mutations that limit their efficacy.

Finally, our study indicates that the effect of drugs targeting mitochondria in overcoming the MDR of FBXW7-deficient cells is associated with their capacity to activate the ISR. This data is consistent with the fact that mitochondrial stress triggers an ATF4dependent stress response in mammalian cells ${ }^{62}$. Furthermore, the antitumoral effects of antibiotics were also previously associated with the $\mathrm{ISR}^{76}$. Intriguingly, activation of the ISR has also been shown to stimulate mitochondrial translation ${ }^{26}$, so that it is possible that the increased mitochondrial dependence of FBXW7-deficient cancer cells is secondary to an active ISR. Similarly to mitochondrial targeting drugs, therapies that stimulate the ISR are also being explored in cancer therapy ${ }^{77}$. Despite focused efforts to generate selective activators of the ISR, we here report the surprising discovery that several drugs with distinct targets and mechanisms of action are activators of the ISR. This raises the question as to what extent the antitumoral effects of these drugs might be partly due to their effects on the ISR, and leads us to propose that this phenotype should be routinely analyzed when developing new anticancer therapies. Consistent with our findings, a recent study has identified that several ATP-competitive kinase inhibitors directly bind and activate GCN2, thereby activating the $\mathrm{ISR}^{78}$. To what extent activating the ISR is a general strategy to overcome MDR regardless of FBXW7 is also an important question to be addressed in future studies. 


\section{ACKNOWLEDGEMENTS}

We would want to thank Drs. Daniela Hühn and Andrés López-Contreras for insightful comments on the manuscript, as well as Javier Muñoz and Eduardo Zarzuela for their help with proteomic analyses. Research was funded by grants from the Spanish Ministry of Science, Innovation and Universities (RTI2018-102204-B-I00, co-financed with European FEDER funds) and the Spanish Association Against Cancer (AECC; PROYE20101FERN) to OF; from the Spanish Ministry of Science, Innovation and Universities (RTI2018-097596-B-I00, (AEI/10.13039/501100011033 MCI/FEDER, UE), co-financed with European FEDER funds) to FA; and a PhD fellowship from La Caixa Foundation and the Marie Skłodowska-Curie European Union's Horizon 2020 actions (LCF/BQ/IN17/11620001) to LS.

\section{AUTHOR CONTRIBUTIONS}

L.S. contributed to most experiments and data analyses and to the preparation of the figures. B.N. contributed to experiments on the mechanism of action of tigecycline. S.G., H.T. and F.A. performed most bioinformatic analyses. M.A. provided help in mouse xenograft studies. O.F. supervised the study and wrote the MS.

\section{DECLARATION OF INTERESTS}

The authors declare no competing interests. 


\section{MATERIALS AND METHODS}

\section{Cell culture}

All cells were grown at $37^{\circ} \mathrm{C}$ in a humidified air atmosphere with $5 \% \mathrm{CO}_{2}$ unless specified. mES were grown on gelatine and feeder layers, using DMEM (high glucose) (Invitrogen) supplemented with 15\% knockout serum replacement (Invitrogen), LIF (1000 U/ml), 0.1 $\mathrm{mM}$ non-essential amino acids, $1 \%$ glutamax, and 55mM $\beta$-mercaptoethanol. Wild type mES cells (R1) were obtained from the American Type Culture Collection (ATCC). ES Cas9 clones and loss-of-function libraries were previously generated ${ }^{49}$. Human cancer cell lines HEK-293T, DLD-1 and HeLa cells (ATCC) were cultured in standard DMEM (high glucose) (Sigma, D5796) supplemented with 10\% FBS and 1\% penicillin/streptomycin. A2780 cells (ATCC) were maintained in RPMI 1640 medium (EuroClone, ECM2001L), 10\% FBS and 1\% penicillin/streptomycin. For the analysis of CHOP transcription, DLD-1 cells were infected with pCLX-CHOP-dGFP lentiviruses (Addgene, 71299), single cell isolated, and selected on the basis of expressing dGFP in response to tunicamycin.

\section{CRISPR editing}

To generate Fbxw7 knockout ES and FBXW7-/- DLD-1, HeLa and A2780 cell lines, cells were independently infected with lentiviral supernatants encoding sgRNAs against Fbxw7/FBXW7. Each cell line was also infected with the empty pLentiCRISPR v2 vector (Addgene, 52961) to be used as controls. 48h after infection, cells were selected for three days with $2 \mu \mathrm{g} / \mathrm{ml}$ puromycin (Sigma, P8833). To obtain pure knock-out clones, the pool of cells was single-cell grown, expanded, and expression of FBXW7 was analyzed by WB. The same procedure was followed for the generation of $A B C B 1^{-1-}$ and $M C L 1^{-1-}$ cells.

\section{CRISPR-Cas9 screens}

For each screen, $5 \cdot 10^{6}$ cells (50X library coverage) from previously described loss-offunction $\mathrm{mES}$ libraries ${ }^{49}$ were plated on gelatin. Cells were treated for approximately 10 days with the different compounds at doses in which no wild-type ES cells survive. For the UV-light screen, a single UV-light (254-nm UV-C) exposure was performed using a 
UVC 500 UV Crosslinker (Hoefer). Once there were less than 100 resistant clones, these were picked, isolated, and expanded. The resistance of individual clones was validated with the corresponding compound before following to the sequencing step. When the number of resistant clones exceeded 100, a pool of cells was grown, its resistance validated, and then the abundance of sgRNAs in the pool was quantified by PCR followed by next-generation sequencing. To identify the sgRNA sequences inserted in the singleisolated resistant clones, DNA was extracted and the fragment flanking the U6-sgRNA cassette was amplified by PCR and sequenced by Sanger sequencing. To identify the sgRNAs present in a pool of cells, DNA was extracted using a Gentra Puregene Blood Kit (Quiagen, 158445), following the manufacturer's instructions. The U6-sgRNA cassette was then amplified by PCR using the KAPA HIFI Hot Start PCR kit (Roche, KK2502) and different tagged primers required for the subsequent Illumina sequencing. The PCR product was precipitated with sodium acetate $3 \mathrm{M}$ in $\mathrm{EtOH} 100 \%$ at $-80^{\circ} \mathrm{C}$ for at least 20 min, pelleted and resuspended in water prior purification in agarose gel. Following a purity check of the PCR product, samples were sent for Illumina sequencing. sgRNA sequences were extracted from fastq files using Galaxy (https://usegalaxy.org/).

\section{Plasmids}

The lentiviral plasmids pLentiCRISPR v2 (Addgene, 52961) and Lentiguide mCherry (kind gift of Cristina Mayor-Ruiz) were used to express sgRNAs in cells as described ${ }^{79}$. sgRNA sequences were designed using the MIT CRISPR design tool (http://www.genomeengineering.org/crispr/) and are available at Table S1. The lentiviral plasmid FUGWeGFP (Addgene, 14883) was used to constitutively express eGFP. For RUBY3 expression, the eGFP sequence of the FUGW-eGFP vector was replaced with the RUBY3 cDNA ${ }^{80}$. The lentiviral plasmid pCLX-CHOP-dGFP (Addgene, 71299) was used to monitor CHOP transcription levels.

\section{Lentiviral production}

Lentiviral vectors were individually co-transfected with third generation packaging vectors in HEK293T cells, using Lipofectamine 2000 (Invitrogen) to generate viral supernatants 
as previously described ${ }^{81}$. Lentiviral supernatants were collected $36 \mathrm{~h}$ after transfection, pooled and passed through a $0.45 \mu \mathrm{M}$ filter to eliminate cellular debris.

\section{RNA interference}

Exponentially growing cells were trypsinised and transfected in suspension with 50nM of control siRNAs or human siRNAs targeting C-MYC (Horizon Discovery Biosciences, ONTARGETplus siRNAs), following manufacturer's instructions and using Lipofectamine RNAiMAX reagent (Thermo Fisher Scientific) and OPTIMEM medium (Life Technologies). For esiRNA libraries targeting mitochondrial factors (Sigma, MISSION® esiRNA, Table S2), the same protocol was followed with 20nM of esiRNA and in a 96well-plate format.

\section{Compounds}

Compounds used in this study are indicated in Table \$3 and were used at the doses indicated in the Figure Legends. All compounds were dissolved in DMSO except cisplatin and oxaliplatin, which were dissolved in DMF; rapamycin and chloramphenicol, in EtOH; and doxycycline and minocycline, dissolved in sterile water. For the chemical screen we used a previously published in-house chemical library composed of 114 FDA-approved or in clinical trials anti-tumoral drugs solved in $\mathrm{DMSO}^{82}$. The library covers $80 \%$ of the pathways described in Reactome. The number of inhibitors for each pathway was the following: cell cycle (2), cell-cell communication (10), cellular response to external stimuli (11), chemotherapeutics (2), DNA repair (2), extracellular matrix reorganization (3), gene expression (12), hemostasis (16), immune system (20), metabolism proteins (2), organelle biogenesis and maintenance (1), and signal transduction (26).

\section{Flow Cytometry}

For the analysis of mixed populations of cells expressing fluorescent proteins, cells at the corresponding mixture ratios were plated in 6-well tissue culture plates. The following day (or $8 \mathrm{~h}$ after plating for mES), cells were treated with the indicated concentrations of drugs for $72 \mathrm{~h}$ (unless specified), and then analysed by Flow Cytometry. For the analysis of 
CHOP transcription, DLD-1 cells expressing a CHOP-dGFP reporter cells were infected at $50 \%$ with Lentiguide mCherry vectors containing validated sgRNAs against the ISR kinases or an empty vector. 96h post-infection, cells were exposed to the indicated drugs for $72 \mathrm{~h}$. Cells were trypsinised, centrifuged, resuspended in PBS and incubated with DAPI for 10 minutes, and subsequently analysed the different cell populations using a flow cytometer BD FortessaTM (BD Biosciences).

For the analysis of mixed populations of cells expressing fluorescent proteins by HighThroughput flow cytometry, 4.000 cells were seeded in $\mu$ CLEAR bottom 96-well plates (Greiner Bio-One). The following day (or $8 \mathrm{~h}$ for $\mathrm{mES}$ ), cells were treated with the indicated concentrations of drugs for $72 \mathrm{~h}$ (unless otherwise specified), and analysed by HighThroughput Flow Cytometry. For esiRNAs experiments, 4.000 cells were transfected in $\mu$ CLEAR bottom 96-well plates (Greiner Bio-One). Every 3-4 days, a fraction of the culture was analysed by High-Throughput Flow Cytometry, while re-transfecting the rest with the esiRNAs. For all analyses, mixtures of cells were trypsinised, stained with DAPI, and the expression of the different fluorescent markers was analysed by using a BD FACS Canto II ${ }^{\mathrm{TM}}$ (BD Biosciences) in High-Throughput mode. Data was processed with the Flow Jo $10^{\mathrm{TM}}$ software to represent each cell population percentage.

\section{Viability assays}

For clonogenic survival assays, 2.000 cells were plated in 6-well tissue culture plates in the corresponding culture medium. The following day, cells were treated with the indicated concentrations of drugs. Cells were maintained with the compounds for 10 days, changing the medium every $2-3$ days, and then fixed and stained with $0.4 \%$ methylene blue in methanol for $30 \mathrm{~min}$. Cell viability was also measured by High-Throughput Microscopy. In brief, 3.000 cells were seeded per well in $\mu$ CLEAR bottom 96-well plates (Greiner Bio-One) and treated with the indicated concentrations of drugs the following day. $72 \mathrm{~h}$ later, cells were fixed with $4 \%$ PFA and permeabilised with $0.5 \%$ Triton X-100, following standard procedures. Cells were subsequently stained with DAPI and images were automatically acquired from each well using an Opera High-Content Screening 
System (Perkin Elmer) or an ImageXpress Pico Automated Cell Imaging System (Molecular Devices). 20x or 10x magnification lenses were used indifferently, and images taken at non-saturating conditions. Images were then segmented using DAPI signals to generate masks that allowed the quantification of nuclei per condition.

\section{Immunofluorescence}

For measuring ATF4 nuclear translocation, 8.000 cells were seeded per well in $\mu$ CLEAR bottom 96-well plates (Greiner Bio-One). The following day, cells were pre-treated for $1 \mathrm{~h}$ with 50nM of ISRIB or DMSO and then treated with the indicated concentrations of drugs for $3 \mathrm{~h}$. Next, cells were fixed with 4\% PFA and permeabilised with $0.5 \%$ Triton X-100, following standard procedures. After blocking for $30 \mathrm{~min}$, plates were stained with an antiATF4 primary antibody overnight (Table S4), followed by an anti-rabbit IgG-488 secondary antibody (Invitrogen, A21441) at 1:400 for $1 \mathrm{~h}$ in RT on the following day. Plates were then stained with DAPI and images automatically acquired using an Opera HighContent Screening System (Perkin Elmer). A 20x magnification lens was used and images were taken at non-saturating conditions. Images were segmented using DAPI signals to generate masks matching cell nuclei, and the nuclear ATF4 intensity per cell was measured. For mitochondrial analyses, 8.000 cells were seeded per well in $\mu$-slide 8-well plates (Ibidi). The following day, cells were fixed with 4\% PFA and permeabilised with $0.5 \%$ Triton X-100, following standard procedures. After blocking for $30 \mathrm{~min}$, cells were stained with an anti-citrate synthetase (CS) primary antibody for $30 \mathrm{~min}$ at $37^{\circ} \mathrm{C}$ (Table S4), and then with an anti-rabbit lgG-488 secondary antibody (Invitrogen, A21441) at 1:400 for another $30 \mathrm{~min}$ at $37^{\circ} \mathrm{C}$. Cells were then stained with DAPI and images were acquired using a LEICA SP5 WLL confocal microscope. A 63x magnification lens was used and images were taken at non-saturating conditions. Images were segmented using DAPI and 488 signals to generate masks matching cell nuclei and mitochondria, respectively. 


\section{Western Blotting}

Cell pellets were lysed in 50mM Tris $\mathrm{pH} 7.9$, 8M Urea and 1\% Chaps followed by $30 \mathrm{~min}$ incubation with shaking at $4^{\circ} \mathrm{C}$. For FBXW7 detection, pellets were lysed in $20 \mathrm{mM} \mathrm{HEPES}$ $\mathrm{pH} 7.9,0.4 \mathrm{M} \mathrm{NaCl}, 1 \mathrm{mM}$ EDTA and protease inhibitors, followed by sonication and a 30 min incubation with shaking at $4^{\circ} \mathrm{C}$. NuPAGE LDS (Life Technologies) with $10 \mathrm{mM}$ DTT (Sigma) loading buffer was added to $20-30 \mu \mathrm{g}$ of protein extracts, and samples were denatured for $10 \mathrm{~min}$ at $70^{\circ} \mathrm{C}$. For the detection of OXPHOS complexes, the denaturing step was performed at $50^{\circ} \mathrm{C}$ for $1 \mathrm{~h}$. Samples were run in precast gels and transferred for protein detection by using the corresponding primary antibodies (Table S4). The signal associated to HRP-conjugated secondary antibodies (ThermoFisher, mouse 31430 and rabbit 31460) was quantified using a SuperSignal ${ }^{\mathrm{TM}}$ West Pico PLUS Chemiluminescent Substrate kit (ThermoFisher, 34580) and a ChemiDoc MP Imagine System (BIO-RAD, 1708280).

\section{Mass spectrometry}

Whole cell extract samples from WT and FBXW7-deficient DLD-1 or ES cells (2 biological replicates) were trypsin-digested using S-traps, isobaric-labelled with TMT® 11-plex reagents, desalted using a Sep-Pak $\mathrm{C} 18$ cartridge and dried prior high $\mathrm{pH}$ reverse phase HPLC RP-HPLC pre-fractionation. Peptides were pre-fractionated offline by means of high $\mathrm{pH}$ reverse phase chromatography, using an Ultimate 3000 HPLC system equipped with a sample collector. Fractions were then analyzed by LC-MS/MS by coupling an UltiMate 3000 RSLCnano LC system to a Q Exactive Plus mass spectrometer (Thermo Fisher Scientific). Raw files were processed with MaxQuant (v1.6.0.16). Afterwards, the file was loaded in Prostar83 using the intensity values for further statistical analysis. Differential expression analysis was done using the empirical Bayes statistics limma. Proteins with a p-value $<0.05$ and a $\log _{2}$ ratio higher than 0.27 (ES) or 0.3 (DLD-1) were defined as regulated, and the FDR was estimated to be below $2 \%$ by Pounds. 


\section{RNA-seq}

$F B X W 7^{+/+}$and $F B X W 7^{-/}$DLD-1 cells were treated with DMSO or tigecycline $(10 \mu \mathrm{M})$ for 24h. Total RNA was extracted from cell pellets using the Agilent Absolutely RNA Miniprep Kit following the manufacturer's instructions. The sequencing library was constructed with the QuantSeq 3' mRNA-Seq Library Prep Kit (Lexogen), and approximately 10 million reads were obtained per sample by Illumina sequencing. Differential expression analyses were performed using Bluebee ${ }^{\circledR}$ (Lexogen). For Gene Ontology (GO) analyses, the list of significantly upregulated genes (padj<0.1) was used as an input for the "The Gene Ontology Resource", release 2021-09-01 (http://geneontology.org/) ${ }^{84,85}$. The transcriptional signature induced by tigecycline was also used as input at the Connectivity Map (CMap) Query clue.io tool (https://clue.io/) ${ }^{65}$ to identify drugs with signatures similar to that tigecycline.

\section{Animal studies}

Athymic Nude-Foxn1nu 6-week female mice were acquired from Charles Rivers. 5·106 exponentially-growing $F B X W 7^{+/+}$and $F B X W 7$ DLD-1 cells were trypsinised and resuspended in PBS for injection in the flanks of 8-week mice. 6 days after, mice were randomized into three groups per genotype (six groups in total, 10 mice per group) and treatment was started with $1,5 \mathrm{mg} / \mathrm{kg}$ paclitaxel (in vivo reference in Table S3), $50 \mathrm{mg} / \mathrm{kg}$ of tigecycline (in vivo reference in Table S3) or vehicle via intraperitoneal (i.p.) injection, on a three times per week schedule. Tumours were measured every 2-3 days, and once they reached $1600 \mathrm{~mm}^{3}$ (measures were calculated using the standard formula length $\mathrm{x}$ width $\times 0.5$ ), mice were sacrificed and their tumours extracted. Health status of mice was monitored daily. Mice were maintained under standard housing conditions with free access to chow diet and water, as recommended by the Federation of European Laboratory Animal Science Association. All mice work was performed in accordance with the Guidelines for Humane Endpoints for Animals Used in Biomedical Research, and under the supervision of the Ethics Committee for Animal Research of the "Instituto de Salud Carlos III". 


\section{Bioinformatic analyses}

\section{Drug resistance}

Drug responses associated with wild type and $F B X W 7$ mut cancer cell lines from the $\mathrm{NCl}-$ 60 and the CCLE collections were extracted and downloaded from the Genomics and Drugs integrated Analysis (GDA) (http://gda.unimore.it/) portal. The same analysis was performed for wild type and $A B C B 1^{\text {mut }}$ cell lines of the $\mathrm{NCl} 60$ (there was no data associated with $A B C B 1$ mutations available in the CCLE). The linear model analysis between FBXW7 expression levels and the Area Under the Curve (AUC) associated with drugs was performed using the CTRP (https://portals.broadinstitute.org/ctrp.v2.1/) portal. The plot represents the coefficient of the compounds which reached statistical significance. A R version compatible with version 3.6.3 was employed for analysis and representation of data.

\section{GDC Pan-Cancer survival data}

The UCSC XenaBrowser (https://xenabrowser.net/) was used to explore the GDC PanCancer database containing data on patients' survival, drug treatment and tumour FBXW7 gene expression. The data was downloaded, and separated into two datasets: one containing data from patients under treatment and the other from patients without drug treatment information. For each of these datasets, patient's data was stratified into two groups according to the median of $F B X W 7$ expression, and survival data from each group was plotted. A Cox regression, including tumour type as co-variable, was also performed. $\mathrm{R}$ version compatible with version 3.6.3 was employed for analysis and representation of data.

\section{CCLE proteomics}

For proteomic analyses of CCLE, data was extracted from a recently published work ${ }^{86}$. 388 cancer cell lines were classified according to FBXW7 mutational and copy number variation status. Only cell lines harbouring coding, damaging, or non-conserving alterations in $F B X W 7$ were labelled as mutated. Cell lines with an absolute copy number score of 0 for $F B X W 7$ were also included. Differential expression analysis was carried out 
using limma ${ }^{87}$ on normalized expression levels between FBXW7-mutant and WT cancer cell lines. For analysis and representation of the data, $\mathrm{R}$ version 3.6.1 was used. For GSEA analyses of proteomics data, GSEA v2.2.4. was used, using a list of pre-ranked Fold Change values as an input.

\section{Statistics and reproducibility}

No statistical method was used to predetermine sample size. Experiments using cells and mice were randomized. Most experiments were performed three times with two technical replicates per experiment, in two independent mutant clones and either the mean of the three experiments or a representative example is shown in the Figures. Values are reported as the mean $\pm S D$, except for the animal studies, were the mean $\pm S E M$ is indicated. No data were excluded from the analyses. For general assessment of the difference between two sets of data, we used the two-tailed unpaired Student's t-test (GraphPad Prism version 7.04). We tested for statistical significance of the overlap between the two groups of genes using the normal approximation of the exact hypergeometric test (http://nemates.org/MA/progs/overlap_stats.cgi). The computational methods used for the differential expression analyses, GO analyses, GSEA studies and other bioinformatics analyses, are explained in their specific methods section. $\mathrm{n}$. Unless otherwise indicated, threshold FDR and padj values were set at $<0.05$. P-values $<0.05$ were considered to be significant (n.s. $p>0.05,{ }^{*} p<0.05,{ }^{* *} p<0.01,{ }^{* *} p<0.001$ ). All statistical parameters, tests and other relevant detailed information are reported in the Figures and corresponding Figure Legends.

\section{Data availability}

Correspondence and request of materials should be addressed to O.F. RNA sequencing data associated with this work are accessible at the GEO repository, under accession number GSE189499. Mass spectrometry proteomic datasets are available at the PRIDE repository with accession numbers PXD029981. 


\section{FIGURE LEGENDS}

Fig. 1. FBXW7 deficiency leads to multidrug resistance. (A) Representation of the percentage of total cell viability (left $\mathrm{Y}$-axis; blue) and the percentage of $F b x w 7^{-1-} \mathrm{mES}$ cells $48 \mathrm{~h}$ after treatment with 114 different FDA-approved compounds $(5 \mu \mathrm{M})$. The culture started with a mix of $F b x w 7^{+/+}$and $F b x w 7^{--}$mES at a 3:1 ratio. Error bars indicate SD. Cell percentages were quantified by high-throughput flow cytometry. (B) Percentages of $\mathrm{Fbxw} 7^{+/+}$(green) and $\mathrm{Fbxw} 7^{-1}$ (red) mES cells from the experiment defined in (A) with the indicated drugs. (C) Percentage of viable $F B X W 7^{+/+}$(green) and FBXW7/- (red) DLD-1 cells upon treatment with paclitaxel (40nM), vincristine (10nM), doxorubicin (25nM), hydroxyurea $(\mathrm{HU}, 75 \mu \mathrm{M})$, gemcitabine $(10 \mathrm{nM})$, Fluorouracil $(5-\mathrm{FU}, 10 \mu \mathrm{M})$, trametinib $(5 \mu \mathrm{M}), \mathrm{BI} 2536$ (PLK1i, 10nM), pevonedistat (200nM) and rapamycin $(10 \mu \mathrm{M})$ for $72 \mathrm{~h}$. DMSO was used to normalize viability except for rapamycin, for which ethanol was used as a control. DAPI staining was used to count nuclei by high-throughput microscopy. Error bars indicate SD $(n=3) .{ }^{*} p<0.05,{ }^{* *} p<0.01,{ }^{* * *} p<0.001$ (t-test). (D,E) Profile of drug responses in FBXW7mut and $A B C B 1^{\text {mut }}$ cancer cell lines from the $\mathrm{NCI} 60$ (D) and CCLE (E) datasets. Each line represents the response to a specific compound (the higher the score, the less of a response to the compound). No data associated to $A B C B 1$ mutations was available at the CCLE.

Fig. 2. Loss of FBXW7 function is associated to an increased mitochondrial activity. (A) Representation of the $\log _{2} \mathrm{FC}$ values from the proteomic analyses of FBXW7 WT and knockout mES ( $x$-axis) and DLD-1 ( $y$-axis) cells. Known FBXW7 substrates are marked in red. (B) GSEA analysis from the proteomic comparison between $F B X W 7^{+/+}$and FBXW7-1- DLD-1 cells. Normalized enrichment scores (NES), and false discovery rate (FDR) q-values from the most significantly enriched Gene ontology (GO) terms are shown. (C) Preranked GSEA on the genes from the 'Mitochondrial translation' GO term obtained from the proteomic analysis comparing $F B X W 7^{+/+}$and $F B X W 7^{-/-}$DLD-1 cells. The heatmap representation illustrates the overall upregulation of these pathways in FBXW7-deficient cells. (D) GSEA analysis from the proteomic comparison between $\mathrm{Fb} \times W 7^{+/+}$and $\mathrm{Fbxw} 7^{-1-}$ mES cells. Normalized enrichment scores (NES), and false 
discovery rate (FDR) q-values from the most significantly enriched Reactome and Hallmark terms are shown. (E) Preranked GSEA on the genes from the 'Oxidative

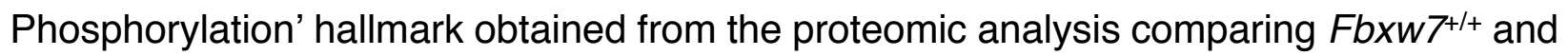
Fbxw $7^{-/-}$mES cells. (F) Representation of the $\log _{2} F C$ values from the proteomic analyses of FBXW7 WT and knockout mES ( $x$-axis) and DLD-1 ( $y$-axis) cells (as in $(A)$ ). Mitochondrial proteins are marked in red. (G) Differential protein expression analysis between $F B X W 7^{+/+}$and $F B X W 7^{\text {mut }}$ cancer cell lines from the CCLE. Proteins significantly upregulated in FBXW7mut cell lines are displayed, with mitochondrial factors highlighted in yellow. (H) GSEA analysis from the proteomic comparison between $F B X W 7^{+/+}$and FBXW7mut cancer cell lines from the CCLE. Normalized enrichment scores (NES), and false discovery rate (FDR) q-values from the most significantly enriched "GO cellular compartment" and "Hallmark" terms are shown. (I) Preranked GSEA on the genes from the 'Oxidative Phosphorylation hallmark obtained from the proteomic analysis comparing $F B X W 7^{+/+}$and $F B X W 7^{\text {mut }}$ cancer cell lines from the CCLE. Note: zero q-values indicate that the value is $<10^{-4}$.

Fig. 3. Targeting mitochondrial function is preferentially toxic for FBXW7-deficient

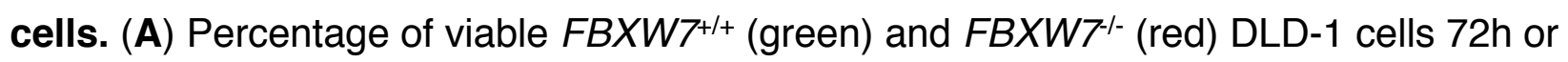
1 week after being treated with the indicated antibiotics. The culture started with a 1:3 ratio of $F B X W 7^{+/+}$and $F B X W 7^{-/-}$cells. The experiment was repeated three times, and a representative example is shown. Error bars indicate SD. n.s. $p>0.05,{ }^{*} p<0.05,{ }^{\star *} p<0.01$, ${ }^{* * *} p<0.001$ (t-test). Cell percentages were quantified by flow cytometry. (B) Normalized percentage of $F B X W 7^{-/-}$cells using increased doses of the indicated antibiotics following the same experimental approach defined in (A). (C) Normalized viability of $F B X W 7^{+/+}$ and FBXW7 $7^{-/}$DLD-1 cells upon treatment with increasing doses of tigecycline for $72 \mathrm{~h}$. Cell nuclei were quantified by high-throughput microscopy (HTM) upon staining with DAPI. The experiment was repeated three times, and a representative example is shown. Error bars indicate SD. (D) Tumour growth (in $\mathrm{mm}^{3}$ ) of $F B X W 7^{+/+}$and $F B X W 7^{-/-}$ xenografts in nude mice ( $n=10$ animals per group). Treatment with either vehicle, paclitaxel $(1,5 \mathrm{mg} / \mathrm{kg})$ or tigecycline $(50 \mathrm{mg} / \mathrm{kg})$ started at day 6 post-tumour-injection, and 
was administered three times per week. Error bars indicate SEM. (E) Representative images of the xenografts defined in (D) at day 15. (F,G) Normalized viability of FBXW7 +/+ and FBXW7 /- DLD-1 cells upon treatment with increasing doses of IACS-10759 (F) or oligomycin (G) for $72 \mathrm{~h}$. Cell nuclei were quantified by HTM as in (C). The experiment was repeated three times, and a representative example is shown. Error bars indicate SD. $(\mathbf{H})$ Percentage of viable $F B X W 7^{+/+}$(green) and $F B X W 7^{-/-}$(red) DLD-1 cells 7 days after being transfected with esiRNAs targeting the indicated mitochondrial factors or luciferase (RLUC) as control. The culture started with a $1: 3$ ratio of $F B X W 7^{+/+}$and $F B X W 7^{-/-}$cells. The experiment was repeated three times, and a representative example is shown. Error bars indicate SD. ${ }^{* *} p<0.01,{ }^{* * *} p<0.001$ (t-test). Cell percentages were quantified by flow cytometry.

Fig. 4. The toxicity of tigecycline is mediated by the ISR. (A) Venn Diagram of the genes that are significantly overexpressed upon a $24 \mathrm{~h}$ treatment with tigecycline $(10 \mu \mathrm{M})$ in $F B X W 7^{+/+}$and FBXW7-- DLD-1 cells (padj <0.1). Note that the antibiotic has a much wider impact on the mutant cells. The p-value indicates the statistical significance of the overlap between the groups. (B) Nuclear ATF4 levels quantified by HTM in $F B X W 7^{+/+}$and FBXW7-/- DLD-1 cells upon treatment with tigecycline $(10 \mu \mathrm{M})$ with or without the ISR inhibitor ISRIB (50nM) for 3h. This experiment was performed 3 times, and a representative example is shown. (D) Clonogenic assays in $F B X W 7^{+/+}$and $F B X W 7^{-/-}$ DLD-1 cells treated with the indicated doses of tigecycline with or without 50nM ISRIB. Control plates were treated with DMSO. This experiment was performed 3 times, and a representative example is shown.

Fig. 5. ISR-activating drugs overcome the MDR of FBXW7-deficient cells. (A) Compounds with a transcriptional signature similar to that triggered by tigecycline identified by CMap using RNAseq data from tigecycline-treated $F B X W 7^{+/+}$and $F B X W 7^{-/-}$ DLD-1 cells. Compound name, compound class and their similarity scores from both genotypes are shown. Mitochondrial poisons (yellow), ISR-activating compounds (green) and additional compounds (blue) are highlighted. (B) Normalized viability of $F B X W 7^{+/+}$ 
and $F B X W 7^{-/-}$DLD-1 cells upon treatment with increasing doses of tunicamycin for $72 \mathrm{~h}$. Cell nuclei were quantified by high-throughput microscopy (HTM) upon staining with DAPI. The experiment was repeated three times, and a representative example is shown. Error bars indicate SD. (C) Nuclear ATF4 levels quantified by HTM in DLD-1 cells upon treatment with the indicated drugs at $10 \mu \mathrm{M}$ (except tunicamycin $(1 \mu \mathrm{M})$ and paclitaxel $(250 \mathrm{nM}))$ for $3 \mathrm{~h}$. This experiment was performed 3 times, and a representative example

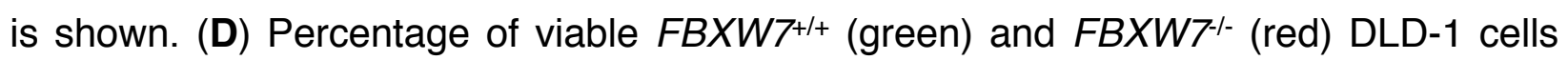
treatment with the indicated drugs at $15 \mu \mathrm{M}$ for $72 \mathrm{~h}$. The culture started with a 1:3 ratio of $F B X W 7^{+/+}$and $F B X W 7^{-/-}$cells. DMSO was used as a control. The experiment was repeated three times, and a representative example is shown. Error bars indicate SD. n.s.: non-significant, ${ }^{* *} p<0.01,{ }^{* * *} p<0.001$ (t-test). Cell percentages were quantified by flow cytometry. (E) Normalized viability of $F B X W 7^{+/+}$and $F B X W 7^{1-}$ cells upon treatment with $10 \mu \mathrm{M}$ of PLX-4720, vemurafenib, erlotinib and gefitinib, and $5 \mu \mathrm{M}$ of sorafenib and dasatinib for $72 \mathrm{~h}$, in the presence or absence of ISRIB (50nM). Cell nuclei were quantified by high-throughput microscopy (HTM) upon staining with DAPI. Error bars indicate SD $(n=3)$. n.s. $p>0.05,{ }^{*} p<0.05,{ }^{\star *} p<0.01,{ }^{* \star *} p<0.001$ (t-test). (F) Heatmap representing of the EGFP median fluorescence intensity (MFI) in DLD-1 cells stably transfected with a reporter construct where a destabilized EFGP is under the control of the human $\mathrm{CHOP}$ promoter, after treatment with the indicated drugs. Cells were infected with lentiviral vectors expressing mCherry and sgRNAs against the four ISR kinases as well or an empty vector. Cell percentages were quantified by flow cytometry. EGFP MFI ratios between mCherry positive and negative cells were first calculated and then normalized to the levels observed in the controls for each treatment. (G) Heatmap representing the viability (calculated as the percentage of mCherry positive cells) in DLD-1 cells treated as in $(\mathbf{F})$. Percentages were normalized first to the DMSO control values and then to the ones found on the empty vector for each treatment. 


\section{REFERENCES}

1 Vasan, N., Baselga, J. \& Hyman, D. M. A view on drug resistance in cancer. Nature 575, 299-309, doi:10.1038/s41586-019-1730-1 (2019).

2 Wang, X., Zhang, H. \& Chen, X. Drug resistance and combating drug resistance in cancer. Cancer Drug Resistance, doi:10.20517/cdr.2019.10 (2019).

3 Zaretsky, J. M. et al. Mutations Associated with Acquired Resistance to PD-1 Blockade in Melanoma. N Engl J Med 375, 819-829, doi:10.1056/NEJMoa1604958 (2016).

$4<$ https://www.cancer.gov/research/key-initiatives/moonshot-cancer-initiative/blue-ribbonpanel\#ui-id-3> (

5 Brockman, R. W. Mechanisms of Resistance to Anticancer Agents. Advances in Cancer Research, 129-234, doi:10.1016/s0065-230x(08)60983-5 (1963).

6 Robert, C. et al. Improved overall survival in melanoma with combined dabrafenib and trametinib. N Engl J Med 372, 30-39, doi:10.1056/NEJMoa1412690 (2015).

7 Mok, T. S. et al. Osimertinib or Platinum-Pemetrexed in EGFR T790M-Positive Lung Cancer. N Engl J Med 376, 629-640, doi:10.1056/NEJMoa1612674 (2017).

8 Shoemaker, R. H., Curt, G. A. \& Carney, D. N. Evidence for multidrug-resistant cells in human tumor cell populations. Cancer Treat Rep 67, 883-888 (1983).

9 Kartner, N., Riordan \& Ling, V. Cell surface P-glycoprotein associated with multidrug resistance in mammalian cell lines. Science 221, 1285, doi:10.1126/science.6137059 (1983).

10 Hata, A. N., Engelman, J. A. \& Faber, A. C. The BCL2 Family: Key Mediators of the Apoptotic Response to Targeted Anticancer Therapeutics. Cancer Discov 5, 475-487, doi:10.1158/2159-8290.CD-15-0011 (2015).

11 Shibue, T. \& Weinberg, R. A. EMT, CSCs, and drug resistance: the mechanistic link and clinical implications. Nat Rev Clin Oncol 14, 611-629, doi:10.1038/nrclinonc.2017.44 (2017).

12 Shen, S., Vagner, S. \& Robert, C. Persistent Cancer Cells: The Deadly Survivors. Cell 183, 860-874, doi:10.1016/j.cell.2020.10.027 (2020).

13 Rehman, S. K. et al. Colorectal Cancer Cells Enter a Diapause-like DTP State to Survive Chemotherapy. Cell 184, 226-242 e221, doi:10.1016/j.cell.2020.11.018 (2021).

14 Farge, T. et al. Chemotherapy-Resistant Human Acute Myeloid Leukemia Cells Are Not Enriched for Leukemic Stem Cells but Require Oxidative Metabolism. Cancer Discov 7, 716-735, doi:10.1158/2159-8290.CD-16-0441 (2017).

15 Farnie, G., Sotgia, F. \& Lisanti, M. P. High mitochondrial mass identifies a sub-population of stem-like cancer cells that are chemo-resistant. Oncotarget 6, 30472-30486 (2015).

16 Ippolito, L. et al. Metabolic shift toward oxidative phosphorylation in docetaxel resistant prostate cancer cells. Oncotarget 7, 61890-61904, doi:10.18632/oncotarget.11301 (2016).

17 Cruz-Bermudez, A. et al. Cisplatin resistance involves a metabolic reprogramming through ROS and PGC-1alpha in NSCLC which can be overcome by OXPHOS inhibition. Free Radic Biol Med 135, 167-181, doi:10.1016/j.freeradbiomed.2019.03.009 (2019).

18 Vazquez, F. et al. PGC1alpha expression defines a subset of human melanoma tumors with increased mitochondrial capacity and resistance to oxidative stress. Cancer Cell 23, 287-301, doi:10.1016/j.ccr.2012.11.020 (2013).

19 Hirpara, J. et al. Metabolic reprogramming of oncogene-addicted cancer cells to OXPHOS as a mechanism of drug resistance. Redox biology 25, 101076, doi:10.1016/j.redox.2018.101076 (2019). 
20 Messner, M. et al. Metabolic implication of tigecycline as an efficacious second-line treatment for sorafenib-resistant hepatocellular carcinoma. FASEB J 34, 11860-11882, doi:10.1096/fj.202001128R (2020).

21 Chen, J., Xu, X. \& Fan, M. Inhibition of mitochondrial translation selectively targets osteosarcoma. Biochem Biophys Res Commun 515, 9-15, doi:10.1016/j.bbrc.2019.05.070 (2019).

$22 \mathrm{Xu}, \mathrm{Y}$. et al. ABT737 reverses cisplatin resistance by targeting glucose metabolism of human ovarian cancer cells. Int J Oncol 53, 1055-1068, doi:10.3892/ijo.2018.4476 (2018).

23 Zhang, L. et al. Metabolic reprogramming toward oxidative phosphorylation identifies a therapeutic target for mantle cell lymphoma. Science Translational Medicine 11, eaau1167, doi:10.1126/scitransImed.aau1167 (2019).

24 Lee, K. M. et al. MYC and MCL1 Cooperatively Promote Chemotherapy-Resistant Breast Cancer Stem Cells via Regulation of Mitochondrial Oxidative Phosphorylation. Cell Metab 26, 633-647 e637, doi:10.1016/j.cmet.2017.09.009 (2017).

25 Roesch, A. et al. Overcoming intrinsic multidrug resistance in melanoma by blocking the mitochondrial respiratory chain of slow-cycling JARID1B(high) cells. Cancer Cell 23, 811825, doi:10.1016/j.ccr.2013.05.003 (2013).

26 Vendramin, R. et al. Activation of the integrated stress response confers vulnerability to mitoribosome-targeting antibiotics in melanoma. $J$ Exp Med 218, doi:10.1084/jem.20210571 (2021).

27 Fulda, S., Galluzzi, L. \& Kroemer, G. Targeting mitochondria for cancer therapy. Nat Rev Drug Discov 9, 447-464, doi:10.1038/nrd3137 (2010).

28 Weinberg, S. E. \& Chandel, N. S. Targeting mitochondria metabolism for cancer therapy. Nat Chem Biol 11, 9-15, doi:10.1038/nchembio.1712 (2015).

29 Skrtic, M. et al. Inhibition of mitochondrial translation as a therapeutic strategy for human acute myeloid leukemia. Cancer Cell 20, 674-688, doi:10.1016/j.ccr.2011.10.015 (2011).

30 D'Andrea, A. et al. The mitochondrial translation machinery as a therapeutic target in Mycdriven lymphomas. Oncotarget 7, 72415-72430 (2016).

31 Riesbeck, K., Bredberg, A. \& Forsgren, A. Ciprofloxacin does not inhibit mitochondrial functions but other antibiotics do. Antimicrobial agents and chemotherapy 34, 167-169, doi:10.1128/aac.34.1.167 (1990).

32 Zhang, L. et al. Antibiotic susceptibility of mammalian mitochondrial translation. FEBS Lett 579, 6423-6427, doi:10.1016/j.febslet.2005.09.103 (2005).

33 Koepp, D. M. et al. Phosphorylation-dependent ubiquitination of cyclin E by the SCFFbw7 ubiquitin ligase. Science 294, 173-177, doi:10.1126/science.1065203 (2001).

34 Yada, M. et al. Phosphorylation-dependent degradation of c-Myc is mediated by the F-box protein Fbw7. EMBO J 23, 2116-2125, doi:10.1038/sj.emboj.7600217 (2004).

35 Wei, W., Jin, J., Schlisio, S., Harper, J. W. \& Kaelin, W. G., Jr. The v-Jun point mutation allows c-Jun to escape GSK3-dependent recognition and destruction by the Fbw7 ubiquitin ligase. Cancer Cell 8, 25-33, doi:10.1016/j.ccr.2005.06.005 (2005).

36 Gupta-Rossi, N. et al. Functional interaction between SEL-10, an F-box protein, and the nuclear form of activated Notch1 receptor. J Biol Chem 276, 34371-34378, doi:10.1074/jbc.M101343200 (2001).

37 Lawrence, M. S. et al. Mutational heterogeneity in cancer and the search for new cancerassociated genes. Nature 499, 214-218, doi:10.1038/nature12213 (2013).

38 Akhoondi, S. et al. FBXW7/hCDC4 is a general tumor suppressor in human cancer. Cancer Res 67, 9006-9012, doi:10.1158/0008-5472.CAN-07-1320 (2007).

39 Yeh, C. H., Bellon, M. \& Nicot, C. FBXW7: a critical tumor suppressor of human cancers. Mol Cancer 17, 115, doi:10.1186/s12943-018-0857-2 (2018). 
$40 \quad$ Kandoth, C. et al. Mutational landscape and significance across 12 major cancer types. Nature 502, 333-339, doi:10.1038/nature12634 (2013).

41 Yan, L. et al. Emerging roles of F-box proteins in cancer drug resistance. Drug Resist Updat 49, 100673, doi:10.1016/j.drup.2019.100673 (2020).

42 Gstalder, C. et al. Inactivation of Fbxw7 Impairs dsRNA Sensing and Confers Resistance to PD-1 Blockade. Cancer Discov 10, 1296-1311, doi:10.1158/2159-8290.CD-19-1416 (2020).

43 Benslimane, Y. et al. Genome-Wide Screens Reveal that Resveratrol Induces Replicative Stress in Human Cells. Mol Cell 79, 846-856 e848, doi:10.1016/j.molcel.2020.07.010 (2020).

44 Hundley, F. V. et al. A comprehensive phenotypic CRISPR-Cas9 screen of the ubiquitin pathway uncovers roles of ubiquitin ligases in mitosis. Mol Cell 81, 1319-1336 e1319, doi:10.1016/j.molcel.2021.01.014 (2021).

45 Liao, S., Maertens, O., Cichowski, K. \& Elledge, S. J. Genetic modifiers of the BRD4-NUT dependency of NUT midline carcinoma uncovers a synergism between BETis and CDK4/6is. Genes Dev 32, 1188-1200, doi:10.1101/gad.315648.118 (2018).

46 Olivieri, M. et al. A Genetic Map of the Response to DNA Damage in Human Cells. Cell 182, 481-496 e421, doi:10.1016/j.cell.2020.05.040 (2020).

47 Wertz, I. E. et al. Sensitivity to antitubulin chemotherapeutics is regulated by MCL1 and FBW7. Nature 471, 110-114, doi:10.1038/nature09779 (2011).

48 Diaz, V. M. \& de Herreros, A. G. F-box proteins: Keeping the epithelial-to-mesenchymal transition (EMT) in check. Semin Cancer Biol 36, 71-79, doi:10.1016/j.semcancer.2015.10.003 (2016).

49 Ruiz, S. et al. A Genome-wide CRISPR Screen Identifies CDC25A as a Determinant of Sensitivity to ATR Inhibitors. Mol Cell 62, 307-313, doi:10.1016/j.molcel.2016.03.006 (2016).

50 Inuzuka, H. et al. SCF(FBW7) regulates cellular apoptosis by targeting MCL1 for ubiquitylation and destruction. Nature 471, 104-109, doi:10.1038/nature09732 (2011).

$51 \mathrm{Li}, \mathrm{N}$. et al. FBXW7-mutated colorectal cancer cells exhibit aberrant expression of phosphorylated-p53 at Serine-15. Oncotarget 6, 9240-9256, doi:10.18632/oncotarget.3284 (2015).

52 Fang, L. et al. Circadian Clock Gene CRY2 Degradation Is Involved in Chemoresistance of Colorectal Cancer. Mol Cancer Ther 14, 1476-1487, doi:10.1158/1535-7163.MCT-150030 (2015).

$53 \mathrm{Li}, \mathrm{N}$. et al. An FBXW7-ZEB2 axis links EMT and tumour microenvironment to promote colorectal cancer stem cells and chemoresistance. Oncogenesis 8, 13, doi:10.1038/s41389-019-0125-3 (2019).

54 Lorenzi, F., Babaei-Jadidi, R., Sheard, J., Spencer-Dene, B. \& Nateri, A. S. Fbxw7associated drug resistance is reversed by induction of terminal differentiation in murine intestinal organoid culture. Mol Ther Methods Clin Dev 3, 16024, doi:10.1038/mtm.2016.24 (2016).

$55 \mathrm{Li}, \mathrm{R}$. et al. miR-223/FBW7 axis regulates doxorubicin sensitivity through epithelial mesenchymal transition in non-small cell lung cancer. Am J Transl Res 8, 2512-2524 (2016).

56 Shoemaker, R. H. The NCl60 human tumour cell line anticancer drug screen. Nat Rev Cancer 6, 813-823, doi:10.1038/nrc1951 (2006).

57 Ghandi, M. et al. Next-generation characterization of the Cancer Cell Line Encyclopedia. Nature 569, 503-508, doi:10.1038/s41586-019-1186-3 (2019). 
58 Basu, A. et al. An interactive resource to identify cancer genetic and lineage dependencies targeted by small molecules. Cell 154, 1151-1161, doi:10.1016/j.cell.2013.08.003 (2013).

59 Grossman, R. L. et al. Toward a Shared Vision for Cancer Genomic Data. N Engl J Med 375, 1109-1112, doi:10.1056/NEJMp1607591 (2016).

60 Molina, J. R. et al. An inhibitor of oxidative phosphorylation exploits cancer vulnerability. Nat Med 24, 1036-1046, doi:10.1038/s41591-018-0052-4 (2018).

61 Costa-Mattioli, M. \& Walter, P. The integrated stress response: From mechanism to disease. Science 368, doi:10.1126/science.aat5314 (2020).

62 Quirós, P. M. et al. Multi-omics analysis identifies ATF4 as a key regulator of the mitochondrial stress response in mammals. J Cell Biol 216, 2027-2045, doi:10.1083/jcb.201702058 (2017).

63 Sidrauski, C. et al. Pharmacological brake-release of mRNA translation enhances cognitive memory. eLife 2, e00498, doi:10.7554/eLife.00498 (2013).

64 Sidrauski, C., McGeachy, A. M., Ingolia, N. T. \& Walter, P. The small molecule ISRIB reverses the effects of elF2a phosphorylation on translation and stress granule assembly. elife 4, e05033, doi:10.7554/eLife.05033 (2015).

65 Subramanian, A. et al. A Next Generation Connectivity Map: L1000 Platform and the First 1,000,000 Profiles. Cell 171, 1437-1452 e1417, doi:10.1016/j.cell.2017.10.049 (2017).

66 Davis, R. J. et al. Pan-cancer transcriptional signatures predictive of oncogenic mutations reveal that Fbw7 regulates cancer cell oxidative metabolism. Proc Natl Acad Sci U S A 115, 5462-5467, doi:10.1073/pnas.1718338115 (2018).

$67 \mathrm{Li}, \mathrm{F}$. et al. Myc stimulates nuclearly encoded mitochondrial genes and mitochondrial biogenesis. Mol Cell Biol 25, 6225-6234, doi:10.1128/mcb.25.14.6225-6234.2005 (2005).

68 Singleton, K. R. et al. Melanoma Therapeutic Strategies that Select against Resistance by Exploiting MYC-Driven Evolutionary Convergence. Cell Rep 21, 2796-2812, doi:10.1016/j.celrep.2017.11.022 (2017).

69 Ravà, M. et al. Therapeutic synergy between tigecycline and venetoclax in a preclinical model of MYC/BCL2 double-hit B cell lymphoma. Science Translational Medicine 10, eaan8723, doi:10.1126/scitransImed.aan8723 (2018).

70 Donati, G. et al. Targeting mitochondrial respiration and the BCL2 family in high-grade MYC-associated B-cell Iymphoma. Molecular Oncology n/a, doi:https://doi.org/10.1002/1878-0261.13115 (2021).

71 Whitfield, J. R. \& Soucek, L. The long journey to bring a Myc inhibitor to the clinic. J Cell Biol 220, doi:10.1083/jcb.202103090 (2021).

72 Bonekamp, N. A. et al. Small-molecule inhibitors of human mitochondrial DNA transcription. Nature 588, 712-716, doi:10.1038/s41586-020-03048-z (2020).

73 Schöller, E. et al. Balancing of mitochondrial translation through METTL8-mediated m(3)C modification of mitochondrial tRNAs. Mol Cell 81, 4810-4825.e4812, doi:10.1016/j.molcel.2021.10.018 (2021).

74 Thandapani, P. et al. Valine tRNA levels and availability regulate complex I assembly in leukaemia. Nature, doi:10.1038/s41586-021-04244-1 (2021).

75 Garofano, L. et al. Pathway-based classification of glioblastoma uncovers a mitochondrial subtype with therapeutic vulnerabilities. Nat Cancer 2, 141-156, doi:10.1038/s43018-02000159-4 (2021).

76 Sharon, D. et al. Inhibition of mitochondrial translation overcomes venetoclax resistance in AML through activation of the integrated stress response. Science Translational Medicine 11, eaax2863, doi:10.1126/scitranslmed.aax2863 (2019).

77 Tian, X. et al. Targeting the Integrated Stress Response in Cancer Therapy. Front Pharmacol 12, 747837, doi:10.3389/fphar.2021.747837 (2021). 
78 Tang, C. P. et al. GCN2 kinase activation by ATP-competitive kinase inhibitors. Nat Chem Biol, doi:10.1038/s41589-021-00947-8 (2021).

79 Sanjana, N. E., Shalem, O. \& Zhang, F. Improved vectors and genome-wide libraries for CRISPR screening. Nature Methods 11, 783-784, doi:10.1038/nmeth.3047 (2014).

80 Bajar, B. T. et al. Improving brightness and photostability of green and red fluorescent proteins for live cell imaging and FRET reporting. Scientific Reports 6, 20889, doi:10.1038/srep20889 (2016).

81 Ruiz, S. et al. A high proliferation rate is required for cell reprogramming and maintenance of human embryonic stem cell identity. Curr Biol 21, 45-52, doi:10.1016/j.cub.2010.11.049 (2011).

82 Bejarano, L. et al. Multiple cancer pathways regulate telomere protection. EMBO Mol Med 11, e10292, doi:10.15252/emmm.201910292 (2019).

83 Wieczorek, S. et al. DAPAR \& ProStaR: software to perform statistical analyses in quantitative discovery proteomics. Bioinformatics 33, 135-136, doi:10.1093/bioinformatics/btw580 (2017).

84 Ashburner, M. et al. Gene ontology: tool for the unification of biology. The Gene Ontology Consortium. Nat Genet 25, 25-29, doi:10.1038/75556 (2000).

85 Consortium, G. O. The Gene Ontology resource: enriching a GOld mine. Nucleic Acids Res 49, D325-d334, doi:10.1093/nar/gkaa1113 (2021).

86 Nusinow, D. P. et al. Quantitative Proteomics of the Cancer Cell Line Encyclopedia. Cell 180, 387-402 e316, doi:10.1016/j.cell.2019.12.023 (2020).

87 Ritchie, M. E. et al. limma powers differential expression analyses for RNA-sequencing and microarray studies. Nucleic Acids Res 43, e47, doi:10.1093/nar/gkv007 (2015). 
A

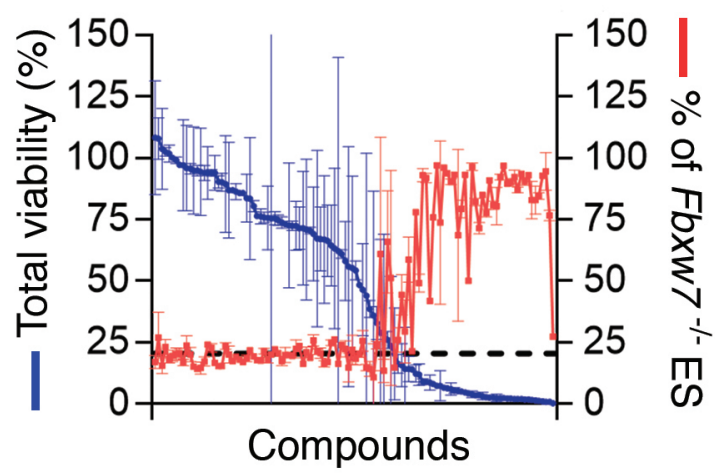

C

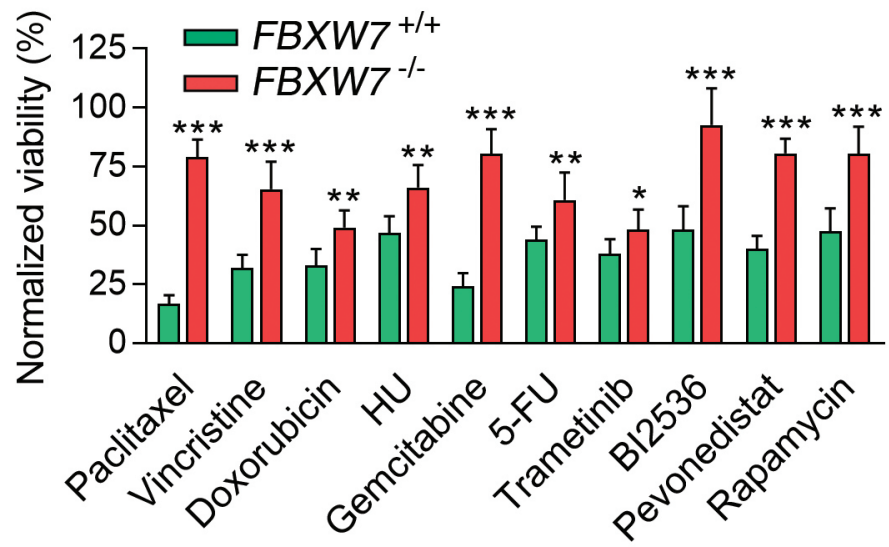

D

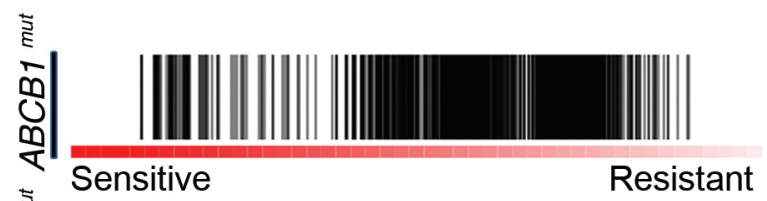

齐

E



B

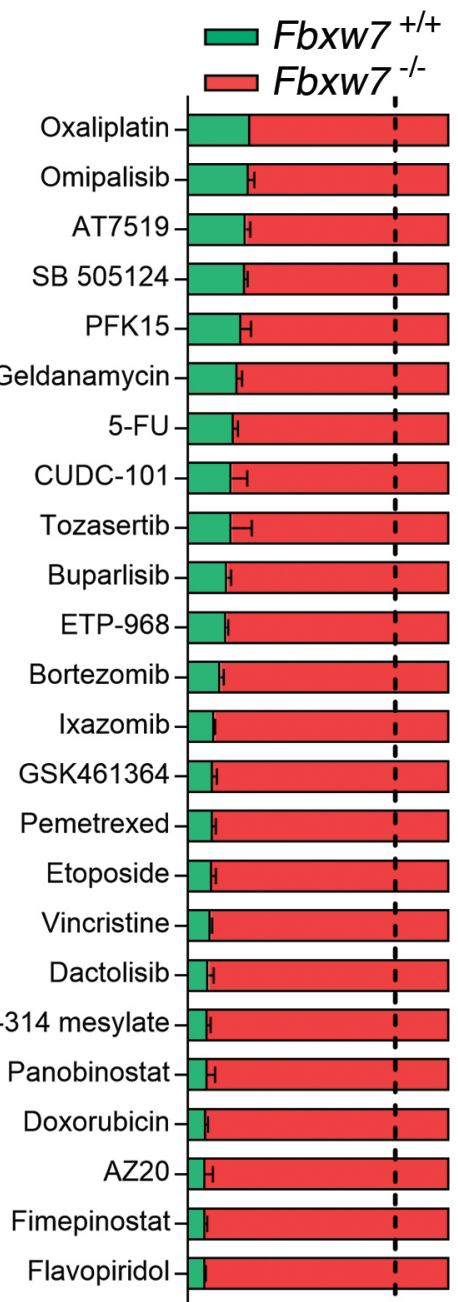

Mitomycin C-

Gemcitabine-

Docetaxel

SN-38-

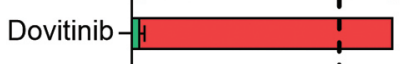

Alisertib -

Vorinostat -

DMSO -

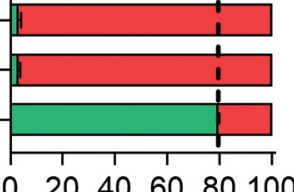

$(\%)$ 
A

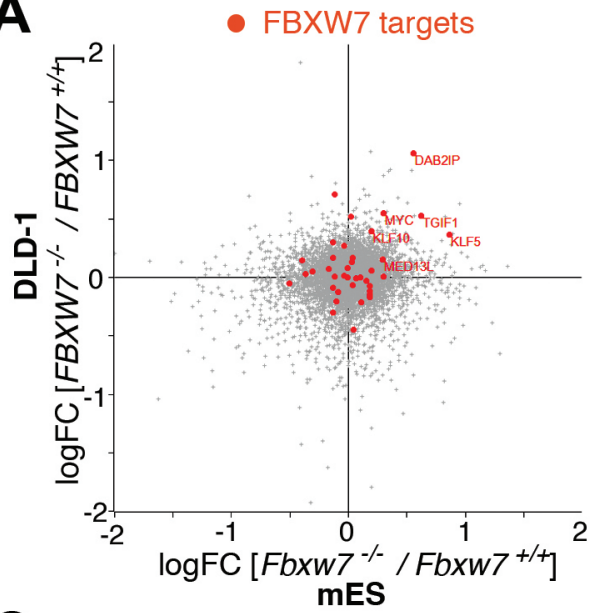

C

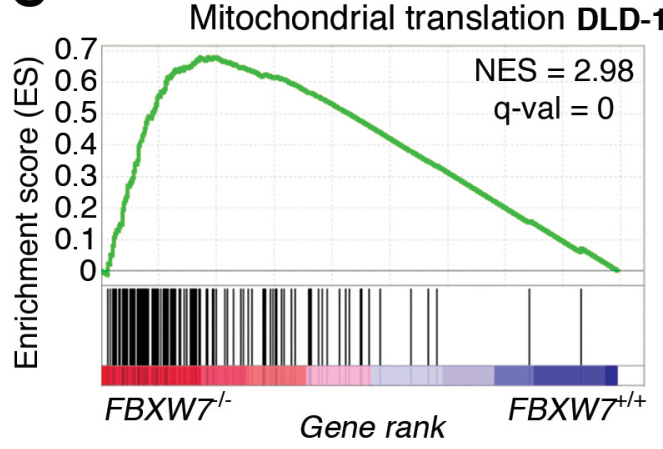

E

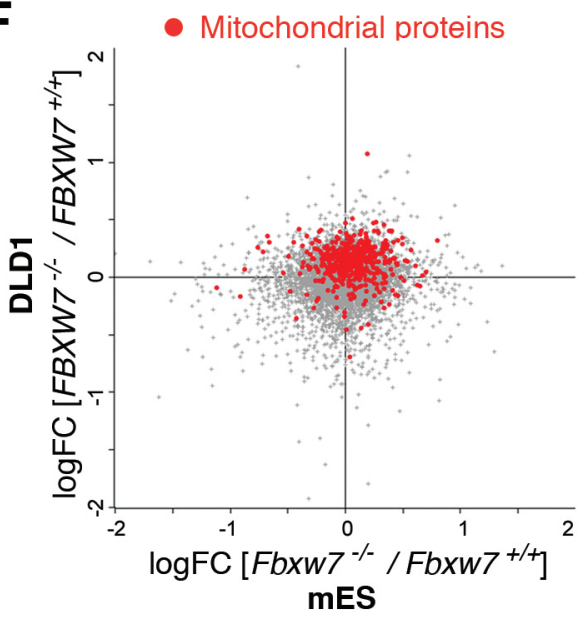

B

\begin{tabular}{|lcc|}
\hline \multicolumn{1}{c}{ GO term } & NES & q-val \\
\hline Mitochondrial translation & 2.98 & 0 \\
Mitochondrial respiratory chain complex assembly & 2.95 & 0 \\
Mitochondrial gene expression & 2.93 & 0 \\
Mitochondrial translation termination & 2.77 & 0 \\
Translation termination & 2.76 & 0 \\
NADH dehydrogenase complex assembly & 2.76 & 0 \\
Oxidative phosphorylation & 2.74 & 0 \\
Cellular respiration & 2.68 & 0 \\
ATP synthesis coupled electron transport & 2.65 & 0 \\
\hline
\end{tabular}

D

\begin{tabular}{|lcc|}
\hline \multicolumn{1}{c}{ Reactome term } & NES & q-val \\
\hline Respiratory electron transport & 2.43 & 0 \\
Respiratory electron transport - ATP synthesis & 2.32 & 0 \\
TCA cycle and respiratory electron transport & 2.03 & 0.006 \\
\hline \multicolumn{1}{|c}{ Hallmark term } & NES & q-val \\
\hline Oxidative phosphorylation & 1.79 & 0.02 \\
\hline
\end{tabular}

E

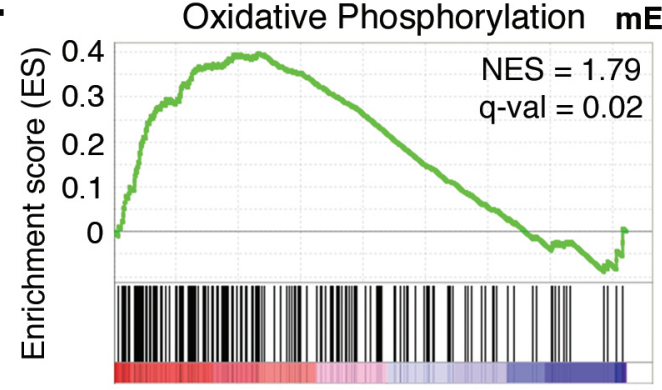

Fbxw $^{\prime-}$

Gene rank
Fbxw $7^{+1+}$

\section{G}

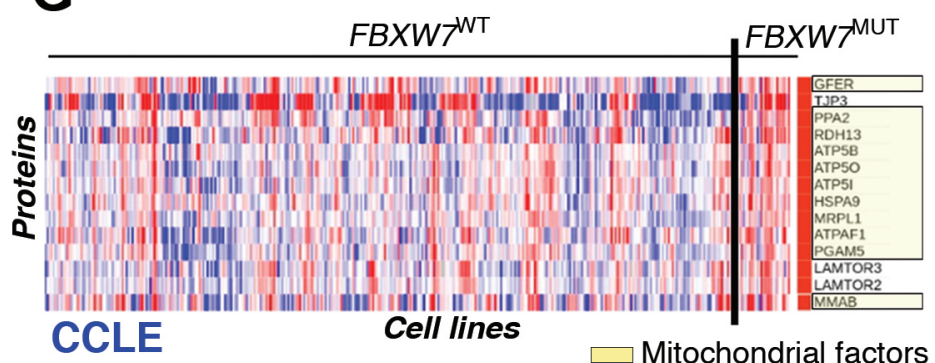

H

\begin{tabular}{lcc|}
\multicolumn{1}{c}{ Pathways enriched in FBXW7 mutant cell lines } \\
\hline \multicolumn{1}{c}{ GO cellular compartment term } & NES & q-val \\
\hline Mitochondrial matrix & 2.80 & 0 \\
Mitochondrial protein containing complex & 2.71 & 0 \\
Organelle inner membrane & 2.71 & 0 \\
Mitochondrial envelope & 2.49 & 0 \\
Inner mitochondrial memb. protein complex & 2.40 & 0 \\
\hline \multicolumn{1}{c}{ Hallmark term } & NES & q-val \\
\hline Oxidative phosphorylation & 2.72 & 0 \\
\hline
\end{tabular}




Sánchez-Burgos et al. Fig 3

A
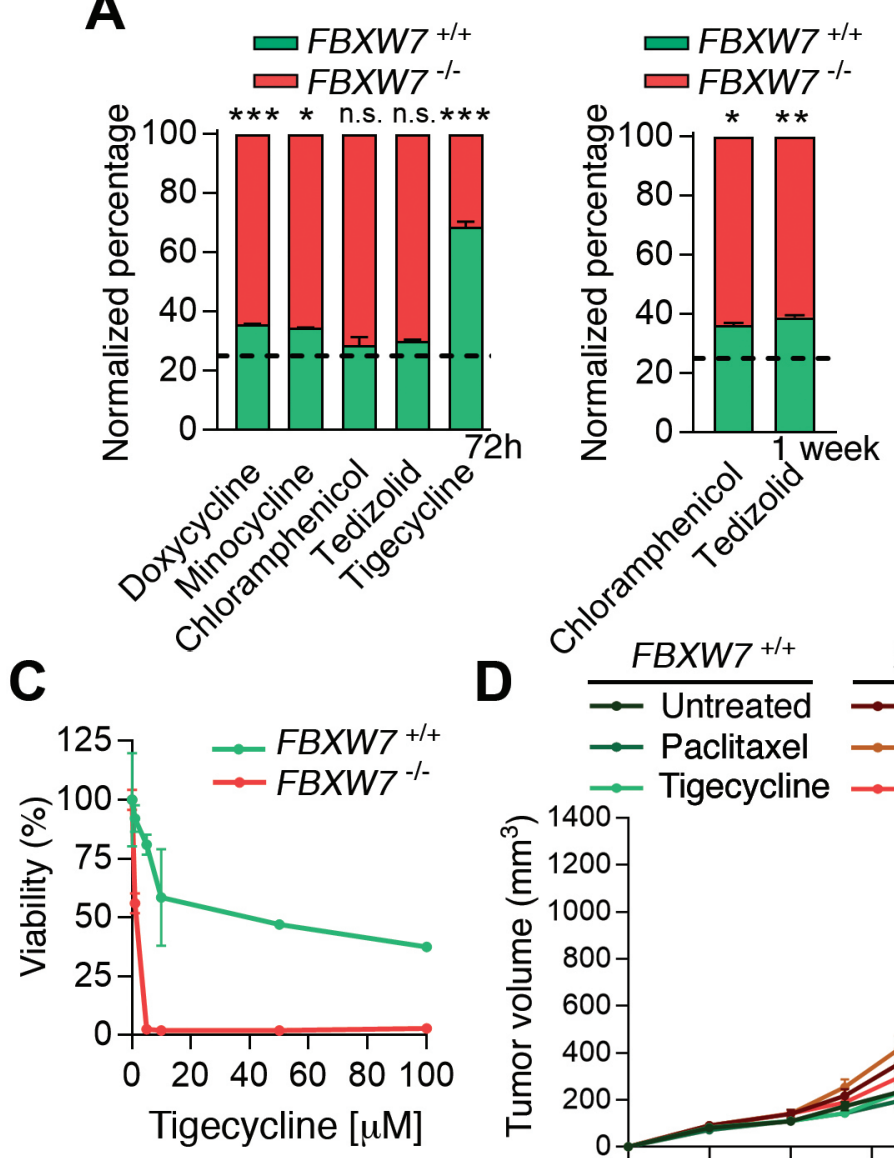

D
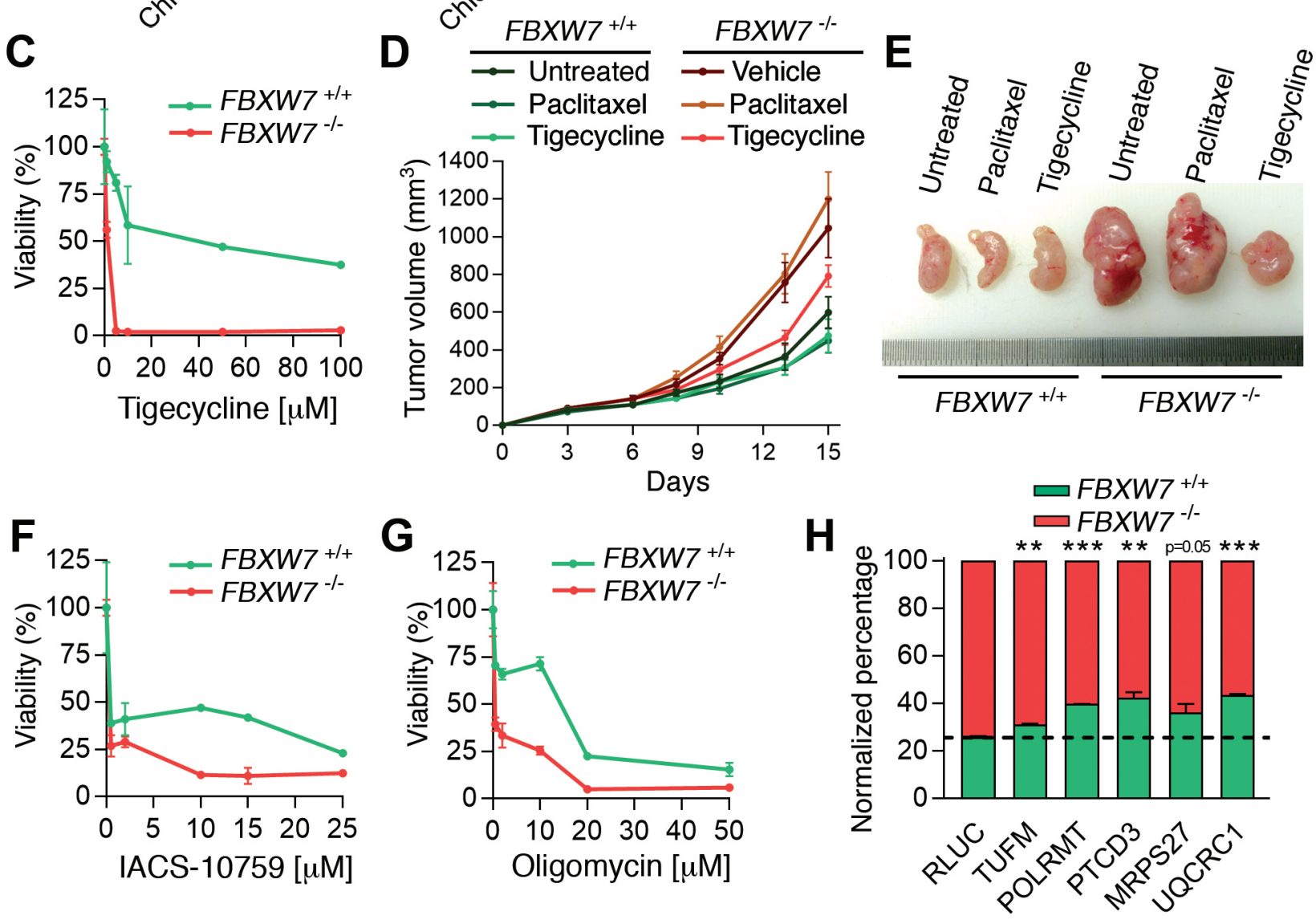

esiRNA 
Sánchez-Burgos et al. Fig 4

A
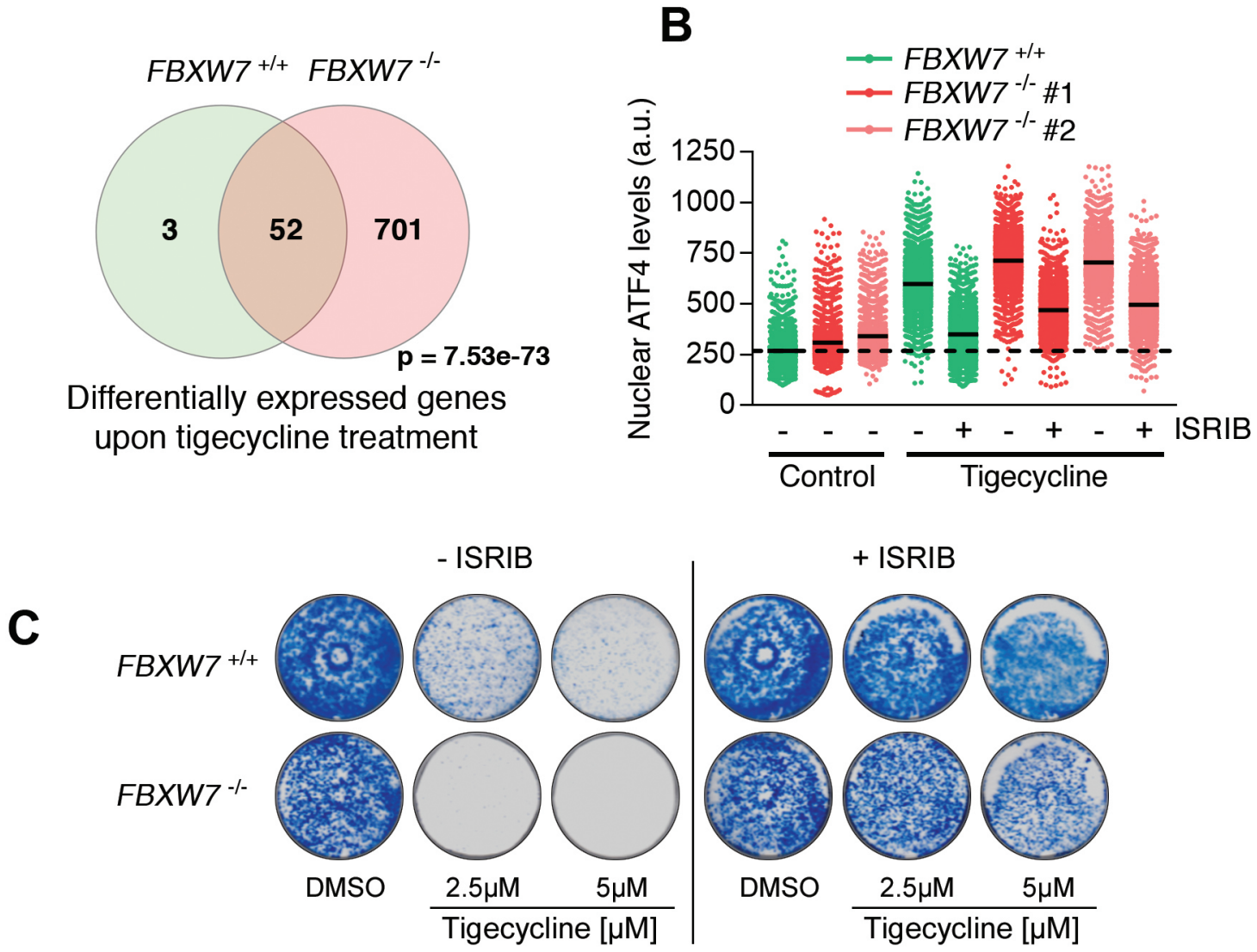
A

Transcriptional signatures similar to tigecycline

\begin{tabular}{llcc}
\hline Compound & Compound Class & $\begin{array}{c}\text { Score } \\
\text { FBXW } 7^{+/+}\end{array}$ & $\begin{array}{c}\text { Score } \\
\text { FBXW } 7^{-1-}\end{array}$ \\
\hline Oligomycin-a & ATP synthase inhibitor & 99,96 & 99,93 \\
\hline Tunicamycin & ER stressor & 99,93 & 99,93 \\
Salubrinal & ISR activator & 99,93 & 99,82 \\
\hline PLX-4720 & RAF inhibitor & 99,86 & 99,08 \\
Vemurafenib & RAF inhibitor & 99,96 & 99,93 \\
Sorafenib & FLT3 inhibitor & 99,93 & 99,93 \\
Dasatinib & BCR-ABL kinase inhibitor & 99,79 & 97,22 \\
Erlotinib & EGFR inhibitor & 99,93 & 99,93 \\
Gefitinib & EGFR inhibitor & 99,86 & 99,44
\end{tabular}

B
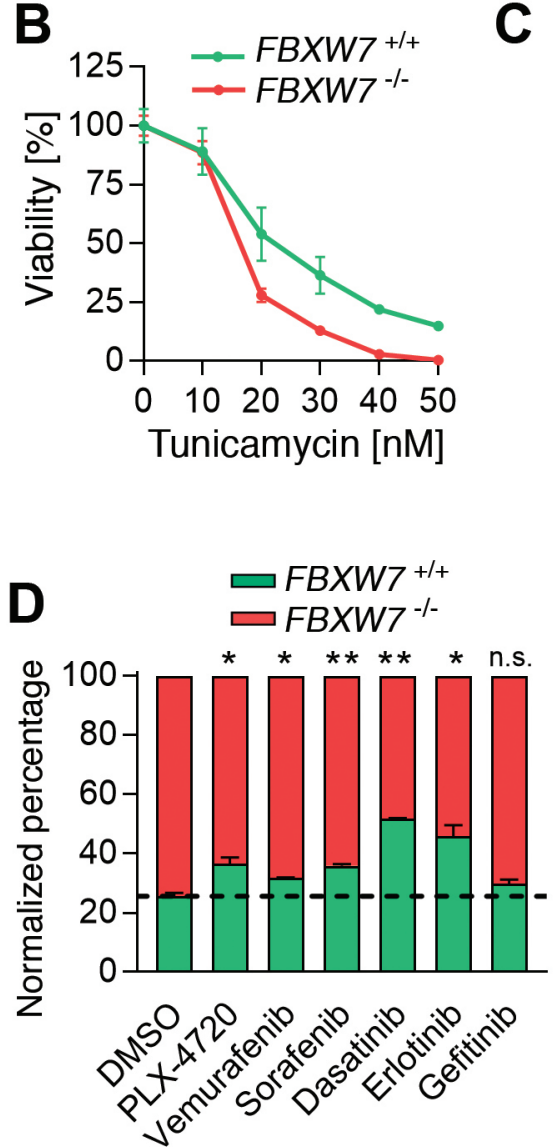

F


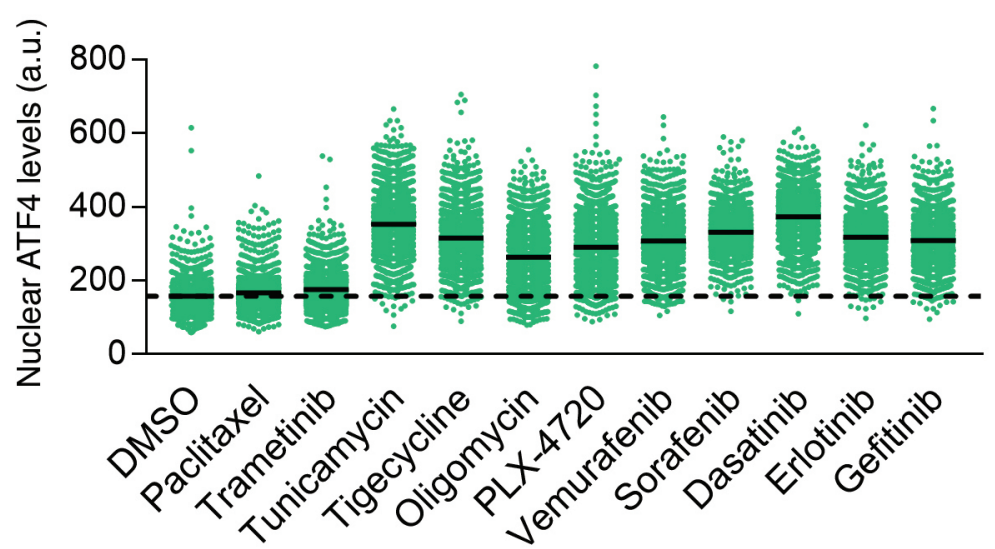

E

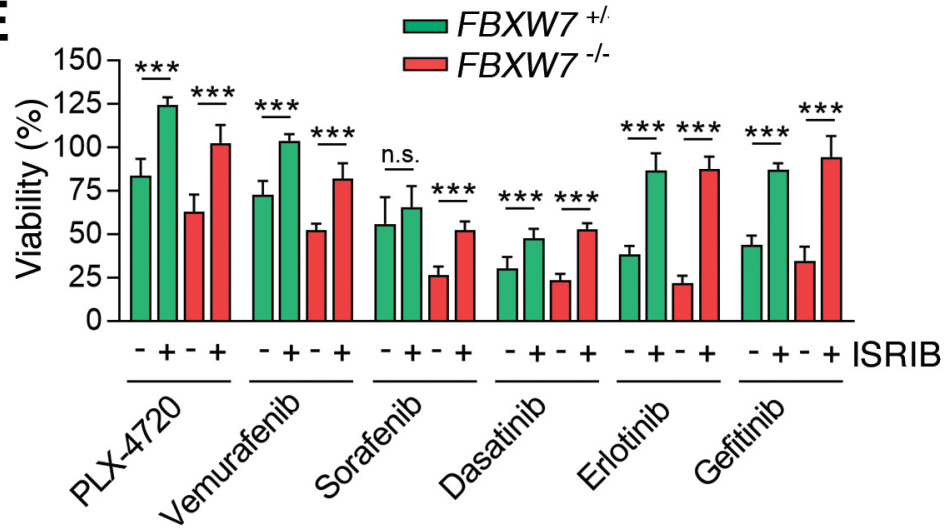

CHOPEGFP $\mathbf{G}$
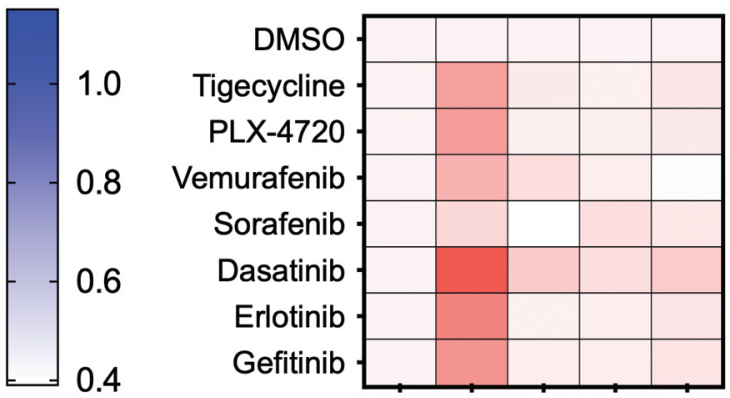

Viability

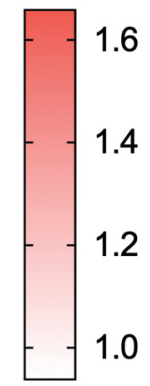

